\title{
Circuits for Voltage Tuning the Parameters of Chua's Circuit: Experimental Application for Musical Signal Generation
}

by GUO-QUN ZHONG†, ROBIN BARGAR $\$$ and KEVIN S. HALLE

Electronics Research Laboratory and Department of Electrical Engineering and Computer Sciences, University of California, Berkeley, CA 94720, U.S.A.

ABSTRACT: It is well known that Chua's circuit can exhibit numerous bifurcation phenomena and attractors by tuning one or more circuit parameters. These properties can be used to synthesize sounds with complex frequency spectra for musical purposes. Investigations of Chua's circuit for sound synthesis and music composition have produced specifications for tuning multiple circuit parameters to obtain specific classes of sounds. This paper presents a multiple control of Chua's circuit in which each parameter of the circuit can be varied by an external control voltage. The design of the subcircuits and the experimental results are shown. Programmable computer software was designed to provide fast multiple-parameter control of the analog C'hua's circuit for use in music composition and live performance. The basic circuit discussed in this paper appeared in a series of live music performances at Expo '93, Seoul, Korea.

\section{Introduction}

The control of chaotic systems is currently a very active research area $(\mathbf{1}, \mathbf{2})$. Several methods for controlling Chua's circuit have been reported (3-8). Most approaches for controlling chaos add an external signal to Chua's circuit to stabilize a periodic orbit, or drive its orbits from a chaotic attractor to its unstable limit cycle, or lock in on a stabilized orbit, and so on. The main goal is to prevent the system from operating in a chaotic regime. In contrast, we propose in this paper a multiple-control approach, for generating a large variety of bifurcation sequences and attractors from Chua's circuit in real time.

The well-known Chua's circuit is a simple electronic chaotic circuit. It has been studied worldwide since its invention in 1983 (9) and its choatic behavior confirmed by computer simulation and experiment, respectively $(\mathbf{1 0}-\mathbf{1 2})$. Chua's circuit and

$\dagger$ On leave from Guangzhou Institute of Electronic Technology, Academia Sinica, Guangzhou 510070, People's Republic of China.

$\ddagger$ National Center for Supercomputing Applications, University of Illinois at UrbanaChampaign. 
its variants (13-18) can exhibit virtually every type of bifurcation and chaotic attractors reported to date in third-order autonomous dynamical systems (19).

By tuning the parameter values of Chua's circuit, various distinct bifurcation sequences and attractors with a rich variety of spectra can be observed (20). These interesting features have been exploited for sound synthesis from an acoustical and musical point of view. It has been discovered by computer simulation $(\mathbf{2 1}, \mathbf{2 2})$ that a rich and novel family of musical sounds can be generated by Chua's circuit, e.g. a period-adding sequence of bassoon-like sounds produces interesting almostharmonic pitch changes, and a time-delayed Chua's circuit can model the basic behavior of an interesting class of musical instruments.

To obtain auditory signals from the circuit, the voltages across two of the energystorage elements, the capacitors, are electronically amplified. To generate musical signals we tune multiple circuit parameters at the same time. A parameter is varied by sending a control signal to the respective circuit component. Programmable computer software has been developed to generate an arbitrary number of simultaneous time-varying digital control signals $(23,24)$. Control signals are transmitted from the computer using a scrial protocol, converted to analog voltages and applied to the circuit (25). The software provides a tool for creating control paths, a method of specifying multiple simultaneous control signals and how they change over time (23). The reproduction of control signals from a control path may be automated or governed by human gestures.

In the multiple-controlled Chua's circuit proposed here, the seven basic parameters can be tuned by external voltages. The circuit operates while the parameters are being controlled in real time by external controlling voltages, regardless of the types of the voltages. There are many combinations of parameters which give rise to a rich variety of bifurcation sequences and chaotic attractors. In Section II, we will describe the design of the controlling circuits. In Section III, we will discuss the programmable software used to compose sound sequences, and the application of the multiple-controlled Chua's circuit to real-time musical performances. In Section IV, we show some examples of bifurcation sequences with respect to some controlled parameters and the corresponding waveforms as well as their spectra

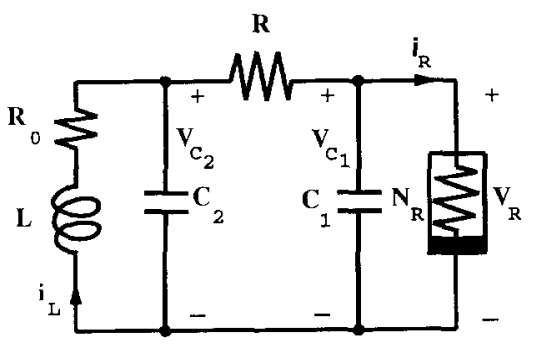

(a)

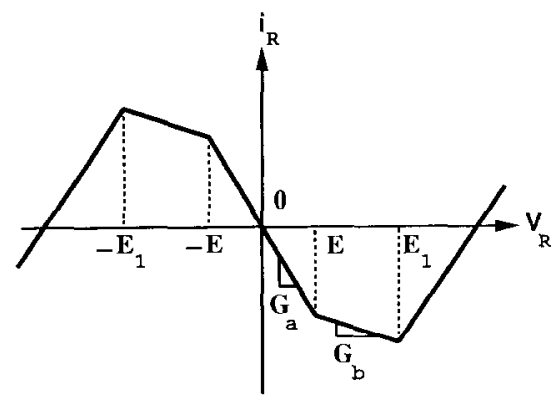

(b)

FIG. 1. (a) Chua's circuit, (b) $v-i$ characteristic of nonlinear resistor $N_{R}$. 
observed experimentally. Some details on the design of subcircuits are presented in the Appendix.

\section{The Design of Multiple-controlled Chua's Circuit}

Figure 1(a) shows a globally unfolded Chua's circuit with a parasitic resistor $R_{0}$. The circuit consists of a linear inductor $L$, two linear capacitors $C_{1}$ and $C_{2}$, two linear resistors $R$ and $R_{0}$, and a nonlinear resistor $N_{R}$ characterized by the piecewiselinear characteristic $i_{R}=g\left(v_{R}\right)$ shown in Fig. 1(b). The state equations for the circuit are

$$
\left.\begin{array}{rl}
\frac{\mathrm{d} v_{c 1}}{\mathrm{~d} t} & =\frac{1}{C_{1}}\left[\frac{1}{R}\left(v_{c 2}-v_{c 1}\right)-g\left(v_{c 1}\right)\right] \\
\frac{\mathrm{d} v_{c 2}}{\mathrm{~d} t} & =\frac{1}{C_{2}}\left[\frac{1}{R}\left(v_{c 1}-v_{c 2}\right)+i_{L}\right] \\
\frac{\mathrm{d} i_{L}}{\mathrm{~d} t} & =\frac{1}{L}\left[-v_{c 2}-R_{0} i_{L}\right]
\end{array}\right\}
$$

In most cases, only the first three segments in Fig. 1(b) are relevant and $g\left(v_{R}\right)$ can be represented by

$$
g\left(v_{R}\right)=G_{b} v_{R}+\frac{1}{2}\left(G_{a}-G_{b}\right)\left[\left|v_{R}+E\right|-\left|v_{R}-E\right|\right] .
$$

In our design of voltage-controlled subcircuits, the key device is an analog multiplier. Here we use AD633JN manufactured by Analog Devices. The connection diagram of AD633JN is shown in Fig. 2. The output of the multiplier is

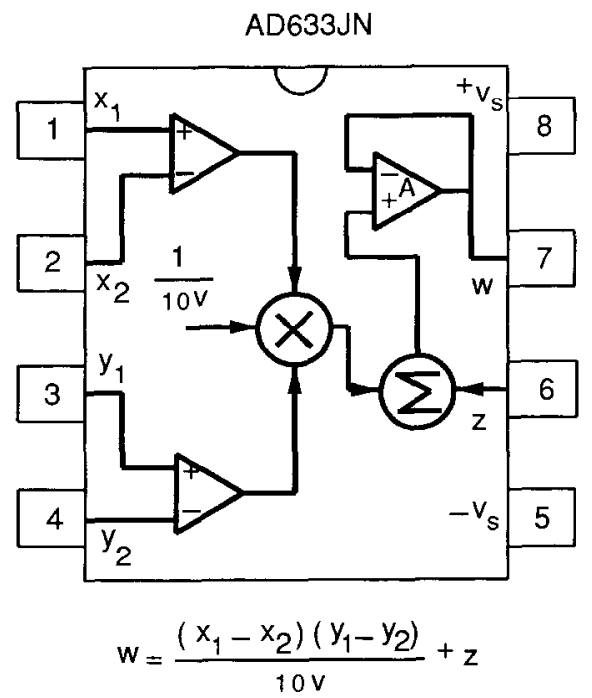

FIG. 2. Connection diagram of multiplier AD633JN. 


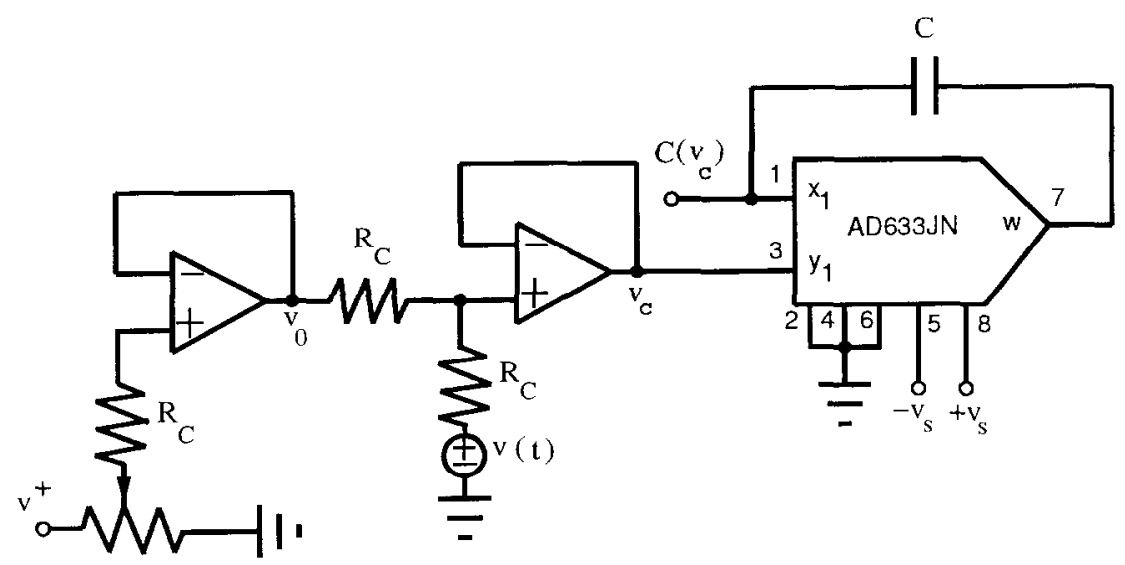

Fig. 3. Schematic of voltage-controlled capacitor circuit.

$$
W=\frac{\left(\begin{array}{ll}
X_{1} & X_{2}
\end{array}\right)\left(\begin{array}{ll}
Y_{1} & Y_{2}
\end{array}\right)}{10 \mathrm{~V}}+Z
$$

\subsection{Voltage-controlled capacitor $C\left(v_{c}\right)$}

A familiar off-the-shelf component, the so-called varactor (variable capacitance diode) is widely utilized in high frequency communication technology. Unfortunately, the capacitance of the typical varactor ranges from several picofarads to several hundred picofarads which is obviously too small for Chua's circuit. The vollage-controlled capacitor which we designed is shown in Fig. 3. The circuit consists of a voltage-controlled capacitor based on the multiplier AD633JN and a voltage controller. From expression (3) and Fig. 3 we obtain (see Appendix) the following expression for $C\left(v_{c}\right)$ (the capacitance between pin 1 of AD633JN and ground):

$$
C\left(v_{c}\right)=C\left(1-\frac{v_{c}}{10 V}\right),
$$

where $v_{c}=\left(v_{0}+v\right) / 2$ since the op amps operate in their linear regions, $v_{0}$ is the offset voltage, $v(t)$ is the external control voltage, and the factor $10 \mathrm{~V}$ is an inherent scaling voltage in the multiplier.

As an example, we pick the recent application used in (25). Here, $C_{1}=14.92$ $\mathrm{nF}, C_{2}=838.1 \mathrm{nF}, v_{0}=0.3 \mathrm{~V}$, and $v_{c} \in\{0.5,2.5\} \mathrm{V}$. Hence $C_{1}\left(v_{c}\right)$ varies from $14.18 \mathrm{nF}$ to $11.20 \mathrm{nF}$, and $C_{2}\left(v_{c}\right)$ varies from $796.8 \mathrm{nF}$ to $628.6 \mathrm{nF}$.

Obviously, it is necessary to include an offset voltage $v_{0}$ in order to set an appropriate range and allow a macro adjustment of the parameter value. Therefore, the voltage controller consists of an offset stage and an adder.

Note in passing that it is convenient to get a desired range of voltage-controlled capacitance with the circuit as long as a relevant externally connected capacitor $C$ is chosen. Here we use the same circuit for the parameters $C_{1}$ and $C_{2}$ in Chua's circuit but with different values of the external capacitance. 


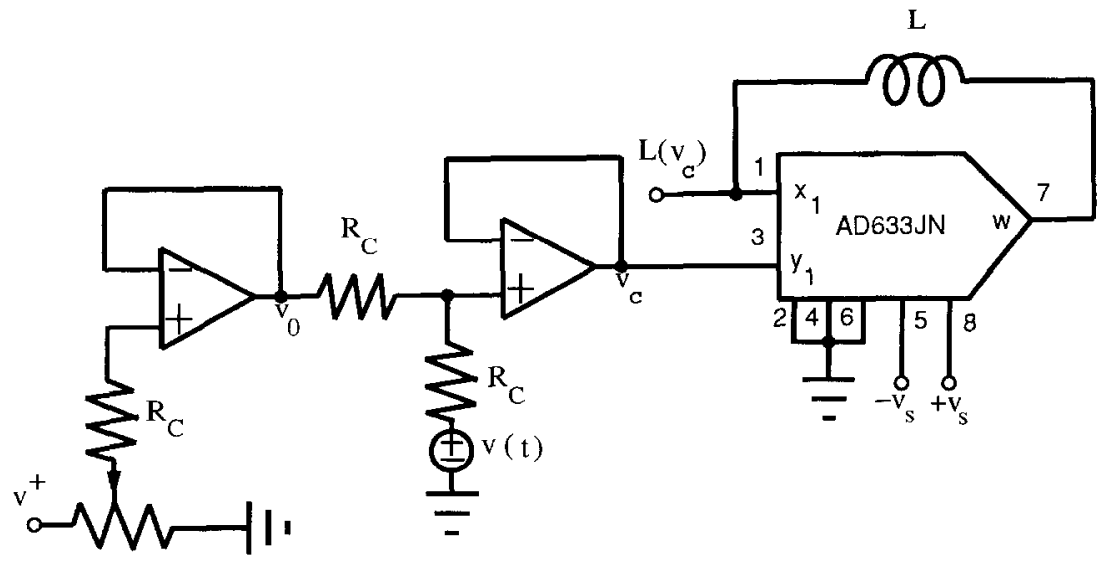

FIG. 4. Schematic of voltage-controlled inductor circuit.

\subsection{Voltage-controlled inductor $L\left(v_{c}\right)$}

By replacing the capacitor $C$ in Fig. 3 with an externally connected inductor $L$, we realize a voltage-controlled inductor $L\left(v_{c}\right)$, as shown in Fig. 4 . By a similar procedure we obtain (see Appendix) the following expression for $L\left(v_{c}\right)$ (the inductance between pin 1 of AD633JN and ground):

$$
L\left(v_{c}\right)=\frac{L}{1-\frac{v_{c}}{10 V}},
$$

where $v_{c}=\left(v_{0}+v\right) / 2$ since the op amps operate in their linear regions, $v_{0}$ is the offset voltage, $v$ is the external control voltage, and the factor $10 \mathrm{~V}$ is the same one as mentioned above.

As an example, we pick the recent application used in (25). Here, $L=38.08 \mathrm{mH}$, $v_{0}=0 \mathrm{~V}$, and $v_{c} \in\{1.371,1.271\} \mathrm{V}$. Hence $L\left(v_{c}\right)$ varies from $44.11 \mathrm{mH}$ to $43.62 \mathrm{mH}$.

Similarly, there is an offset subcircuit for setting an appropriate range and allowing a macro adjustment for $L\left(v_{c}\right)$. By choosing a relevant externally connected inductor $L$, we obtain the desired voltage-controlled inductance.

\subsection{Voltage-controlled resistor $R\left(v_{c}\right)$}

By replacing the capacitor $C$ in Fig. 3 with an externally connected resistor $R$ we realize the voltage-controlled resistor $R\left(v_{c}\right)$ shown in Fig. 5. By a similar procedure we obtain (see Appendix) the following expression for $R\left(v_{c}\right)$ (the resistance between pin 1 of $\triangle \mathrm{D} 633 \mathrm{JN}$ and ground) :

$$
R\left(v_{c}\right)=\frac{R}{1-\frac{v_{c}}{10 \mathrm{~V}}},
$$

where $v_{c}=\left(v_{0}+v\right) / 2$ since the op amps operate in their linear regions, $v_{0}$ is the offset voltage, $v$ is the external control voltage, and the factor $10 \mathrm{~V}$ is the same one as mentioned above. 


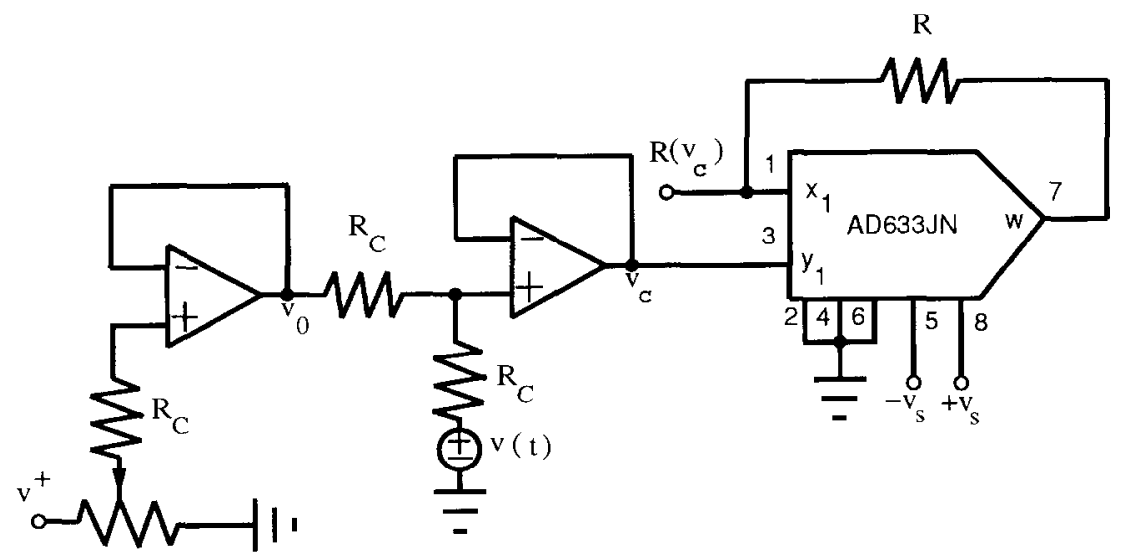

FIG. 5. Schematic of voltage-controlled resistor circuit.

Similarly, there is an offset subcircuit for setting an appropriate range and allowing a macro adjustment for $R\left(v_{c}\right)$. By choosing a relevant externally connected resistor $R$, we obtain the desired voltage-controlled resistance.

We use this voltage-controlled resistor for $R_{0}$ in Fig. 1(a).

As an example, we pick the recent application used in (25). Here, $R_{0}=5.332 \Omega$, $v_{0}=3.131 \mathrm{~V}$, and $v_{c} \in\{2.3,5.0\} \mathrm{V}$. Hence $R_{0}\left(v_{c}\right)$ varies from $11.64 \Omega$ to $14.92 \Omega$.

\subsection{Voltage-controlled resistor with floating terminals}

The resistor $R$ in Chua's circuit shown in Fig. 1(a) needs to be floating above ground. Obviously, the voltage-controlled resistor shown in Fig. 5 does not work in this case. The voltage-controlled resistor with floating terminals above ground which we designed is shown in Fig. 6. The expression for the equivalent resistance between terminals $a$ and $b$ can be obtained by means of similar procedure (see Appendix), i.e.

$$
R\left(v_{c}\right)=\frac{R}{\frac{v_{c}}{10 V}},
$$

where $v_{c}=\left(v_{0}+v\right) / 2$ since the op amps operate in their linear regions, $v_{0}$ is the offset voltage, $v$ is the external control voltage, and the factor $10 \mathrm{~V}$ is the same one as mentioned above.

As an example, we pick the recent application used in (25). Here, $R=682.0 \Omega$, $v_{0}=0.5 \mathrm{~V}$, and $v_{c} \in\{1.5,2.5\} \mathrm{V}$. Hence $R\left(v_{c}\right)$ varies from $1.954 \mathrm{k} \Omega$ to $1.476 \mathrm{k} \Omega$.

Similarly, there is an offset subcircuit for setting an appropriate range and allowing a macro adjustment for $R\left(v_{c}\right)$. Choosing a relevant externally connected resistor $R$, we obtain the desired voltage-controlled resistor with floating terminals.

\subsection{Voltage-controlling breakpoints $+E$ and $-E$}

The breakpoints of the piecewise-linear resistor $N_{R}$ 's $v-i$ characteristic are proportional to the saturation levels of the op amps from which $N_{R}$ is built. The 


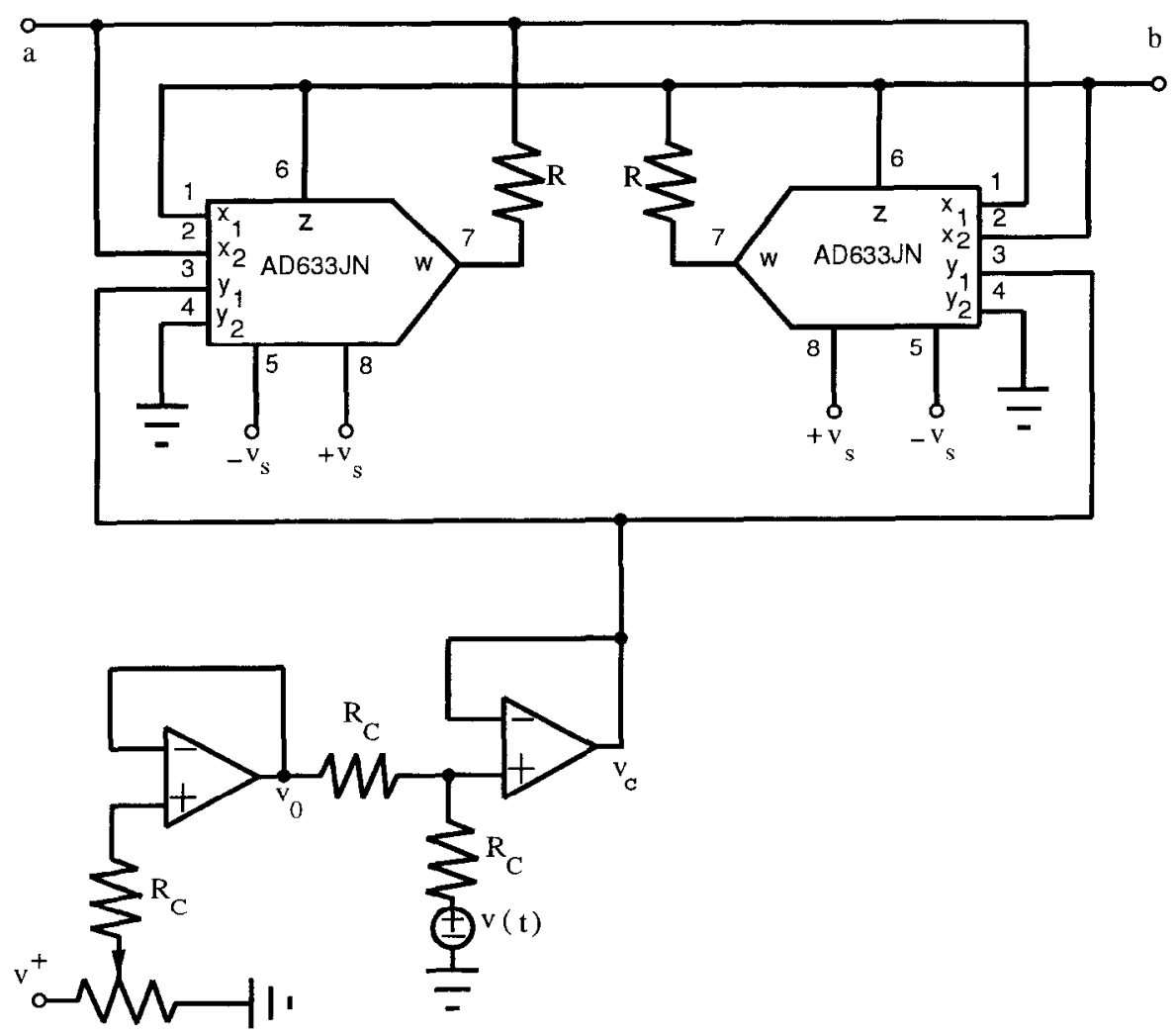

FIG. 6. Schematic of voltage-controlled resistor with floating terminals circuit.

saturation levels are determined by the power supply voltages and the architectures of the op amps. The slopes of the $v-i$ characteristic of $N_{R}$ do not depend on the power supply voltages. Therefore, the idea for controlling the breakpoints of the $v-i$ characteristic is to control the power supply voltages for the op amps used for realizing the piecewise-linear resistor $N_{R}$. This circuit is shown in Fig. 7. We can see that the circuit consists simply of two voltage-controlled voltage sources $+v_{s}$ and $-v_{s}$ that supply the power for the op amps of $N_{R}$. The two breakpoints $+E$ and $-E$ of $N_{R}$ 's $v-i$ characteristic can be controlled separately by this circuit, i.e. $+E$ and $-E$ will be changed by adjusting controlling voltages $v_{1}(t)$ and $v_{2}(t)$ separately.

As an example, we pick the recent application used in (25). Here, $+E=+1 \mathrm{~V}$, $-E=-1 \mathrm{~V}$, when $v_{0}=0 \mathrm{~V},+v_{s}=+5.327 \mathrm{~V},-v_{s}=-5.974 \mathrm{~V}$. Hence $+E\left(v_{c}\right)$ varies from $+0.35 \mathrm{~V}$ to $+0.65 \mathrm{~V}$ for $+v_{s} \in\{2.415,3.847\} \mathrm{V}$, and $-E\left(v_{c}\right)$ varies from $-0.65 \mathrm{~V}$ to $-0.85 \mathrm{~V}$ for $-v_{s} \in\{-4.385,-5.050\} \mathrm{V}$.

\subsection{Voltage-controlling slopes $G_{a}$ and $G_{b}$}

The schematic diagram of the piecewise-linear resistor $N_{R}$ is shown in Fig. 8(a). The $N_{R}$ 's $v-i$ characteristic measured when $R_{1}=R_{2}=220 \Omega, R_{3}=2.4 \mathrm{k} \Omega$, $R_{4}=R_{5}=22 \mathrm{k} \Omega, R_{6}=3.3 \mathrm{k} \Omega$ is shown in Fig. 8(b). From (26) we have 


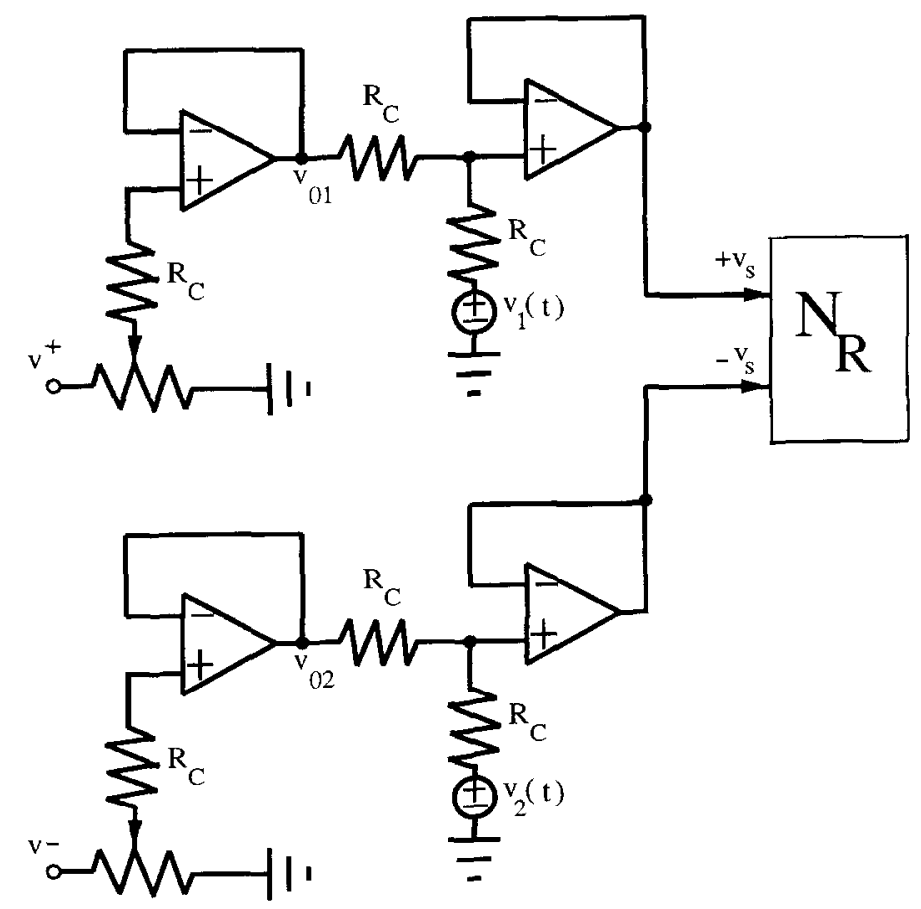

FIG. 7. Schematic of voltage-controlling breakpoints $\pm E$ of $v-i$ characteristic of $N_{R}$.

$$
\left.\begin{array}{l}
G_{a}=m_{11}+m_{12} \\
G_{b}=m_{11}+m_{02} \\
E=\frac{R_{6}}{R_{5}+R_{6}} E_{s a t} \\
E_{1}=\frac{R_{3}}{R_{2}+R_{3}} E_{s a t}
\end{array}\right\}
$$

where

$$
m_{11}=-\frac{1}{R_{3}}, \quad m_{12}=-\frac{1}{R_{6}} \quad \text { with } \quad R_{2}=R_{1}, \quad m_{02}=\frac{1}{R_{4}}
$$

with $R_{5}=R_{4}$, and $E_{s a t}$ is the saturation level of the op amp. Obviously, adjusting $R_{3}$ will change the slopes $G_{a}$ and $G_{b}$ simultaneously and only the outer breakpoints $E_{1}$ which is not very interesting to us here. Introducing a voltage-controlled resistor, shown in Fig. 5, to the piecewise-linear resistor $N_{R}$ for $R_{3}$, the voltage-controlled slopes $G_{a}$ and $G_{b}$ will be realized, as shown in Fig. 8, where $R_{3}\left(v_{c}\right)$ is a voltagecontrolled resistor like that shown in Fig. 5.

As an example, we pick the recent application used in (25). Here, $G_{a}=-0.72$ $\mathrm{mS}, G_{b}=-0.5 \mathrm{mS}$, when $v_{\mathrm{n}}=0 \mathrm{~V},+v_{s}=+5.327 \mathrm{~V},-v_{s}=-5.974 \mathrm{~V}$. Hence $G_{a}\left(v_{c}\right)$ varies from $-0.77 \mathrm{mS}$ to $-0.7 \mathrm{mS}$, and $G_{b}\left(v_{c}\right)$ varies from $-0.51 \mathrm{mS}$ to $-0.45 \mathrm{mS}$ for $v_{c} \in\{5.555,5.856\} \mathrm{V}$, respectively. 
(a)

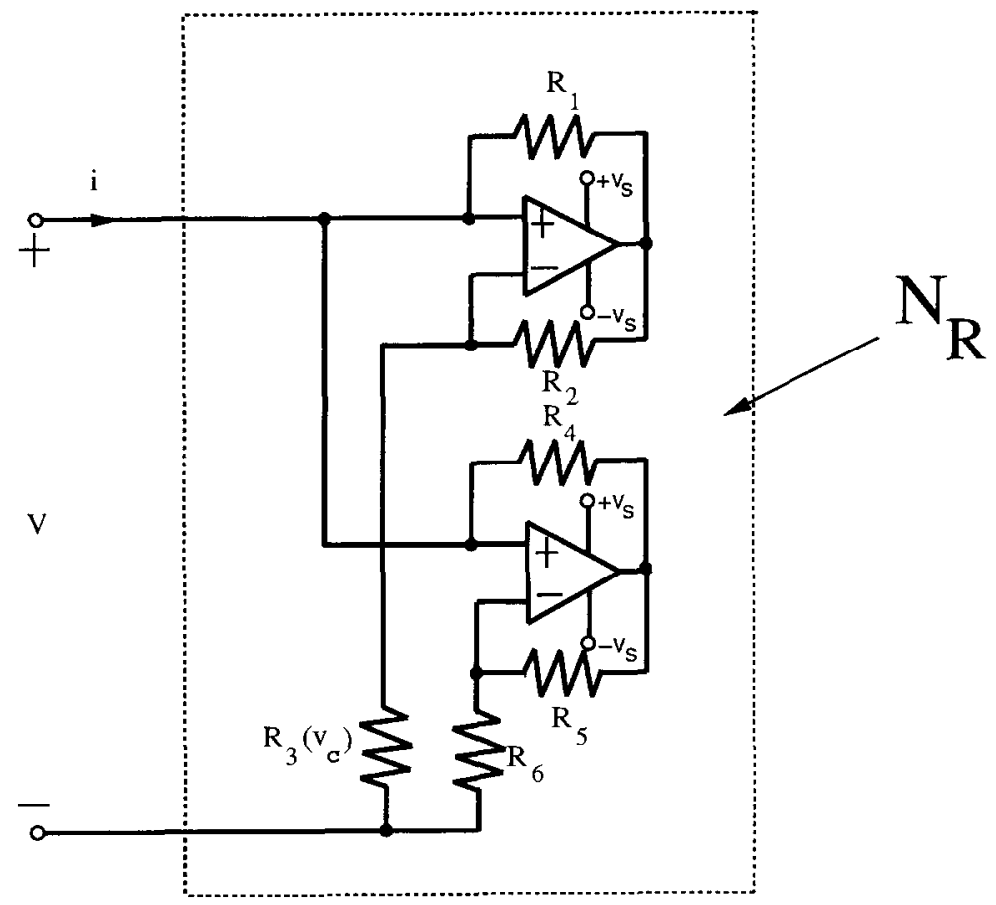

(b)

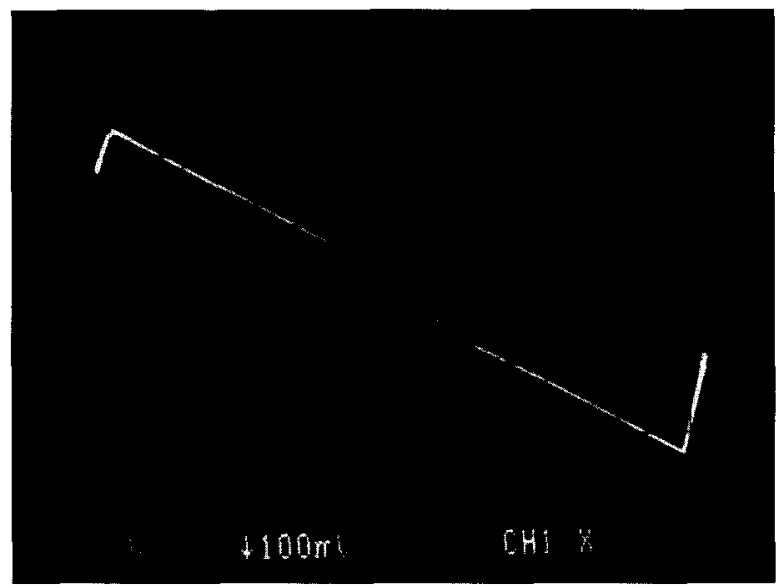

FIG. 8. (a) Schematic of piecewise-linear resistor $N_{R}$, (b) Measured $v-i$ characteristic of piecewise-linear resistor $N_{R}$. Horizontal axis $v$, scale : $1 \mathrm{~V} / \mathrm{div}$; Vertical axis $i$, scale : $1 \mathrm{~mA} / \mathrm{div}$.

Thus, the parameters $C_{1}, C_{2}, L, R, R_{0},+E,-E$, and slopes $G_{a}, G_{b}$, in Chua's circuit can all be adjusted via external controlling voltages.

\subsection{Simultaneous voltage-control of $R, R_{0}$ and slopes $G_{a}, G_{b}$}

Simultaneous variation of several parameters produces unique acoustic transitions which cannot be obtained by tuning a single parameter. Figure $9(\mathrm{a})-(\mathrm{e})$ shows the covariance of $R, R_{0}$, and slope $G_{b}$, and a sequence of the resulting states, 


\section{G.-Q. Zhong et al.}

respectively. Along this path we encounter fixed points, a number of limit cycles and intermittent patterns in regions of unstable periodic limit cycles, as well as chaotic regions. The signals in Fig. 9(b)-(e) were created by applying multiple parameter variation to the circuit while listening to the resulting signal. We refer to this process as tuning the parameters of the Chua's circuit. As an example, we present some waveforms and the corresponding spcctra of a signal generated by the circuit at $t=1 \mathrm{~s}, 4 \mathrm{~s}, 6 \mathrm{~s}$, and $10 \mathrm{~s}$ during the acoustic transition of the path, as shown in Fig. 9(b)-(e), respectively. Note from the figures that a series of spectra with different patterns can be obtained along the path by multiple-controlling the parameters of the circuit. Novel musical signals will be generated based on the control to the circuit.

Comparing the results shown in Fig. 9(b)-(e) with the observations presented in Section IV, it can be noted that a large variety of spectrum patterns can be obtained. In addition, tuning the parameters $R, R_{0}$, and slopes $G_{a}$ and $G_{l}$, changes
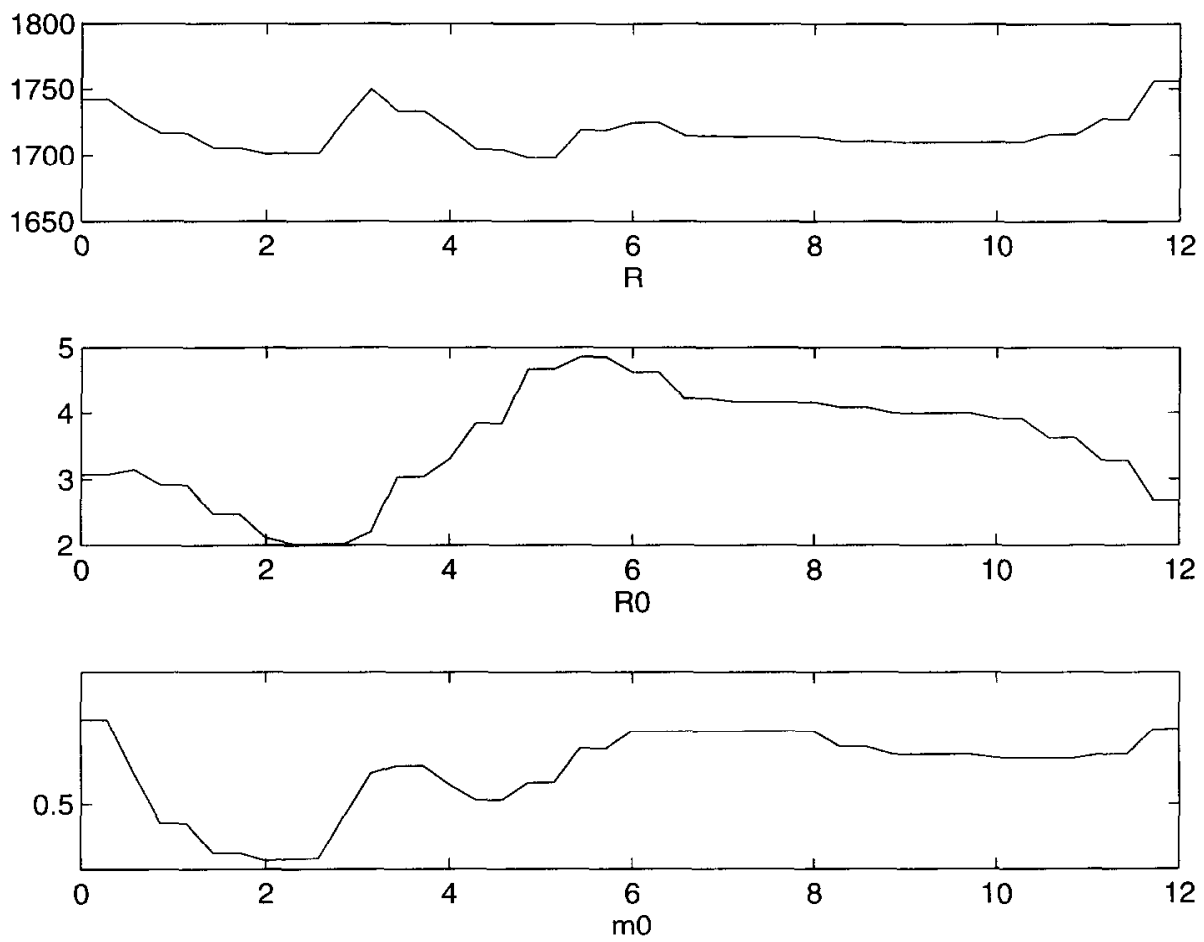

(a)

FIG. 9. The covariance of $R, R_{0}$, and slope $G_{h}$, and a sequence of the resulting states. (a) The covariance of $R, R_{0}$, and slope $G_{b}$; Horizontal axis $t$, unit second ; Vertical axis $R$ (top), $R_{0}$ (mid), slope $G_{b}$ (bottom), unit $\Omega$ for $R$ and $R_{0}-\mathrm{mS}$ for $G_{b}$. (b)-(e) The time waveform (top) and the corresponding spectrum diagram (bottom); Horizontal axis $t$ (top), $f$ (bottom), scale: $2 \mathrm{msec} / \mathrm{div}$ (top), $250 \mathrm{~Hz} / \mathrm{div}$ (bottom); Vertical axis $v_{c t}$, scale : $200 \mathrm{mV} / \mathrm{div}$ (top), $10 \mathrm{~dB} / \mathrm{div}$ (bottom). 

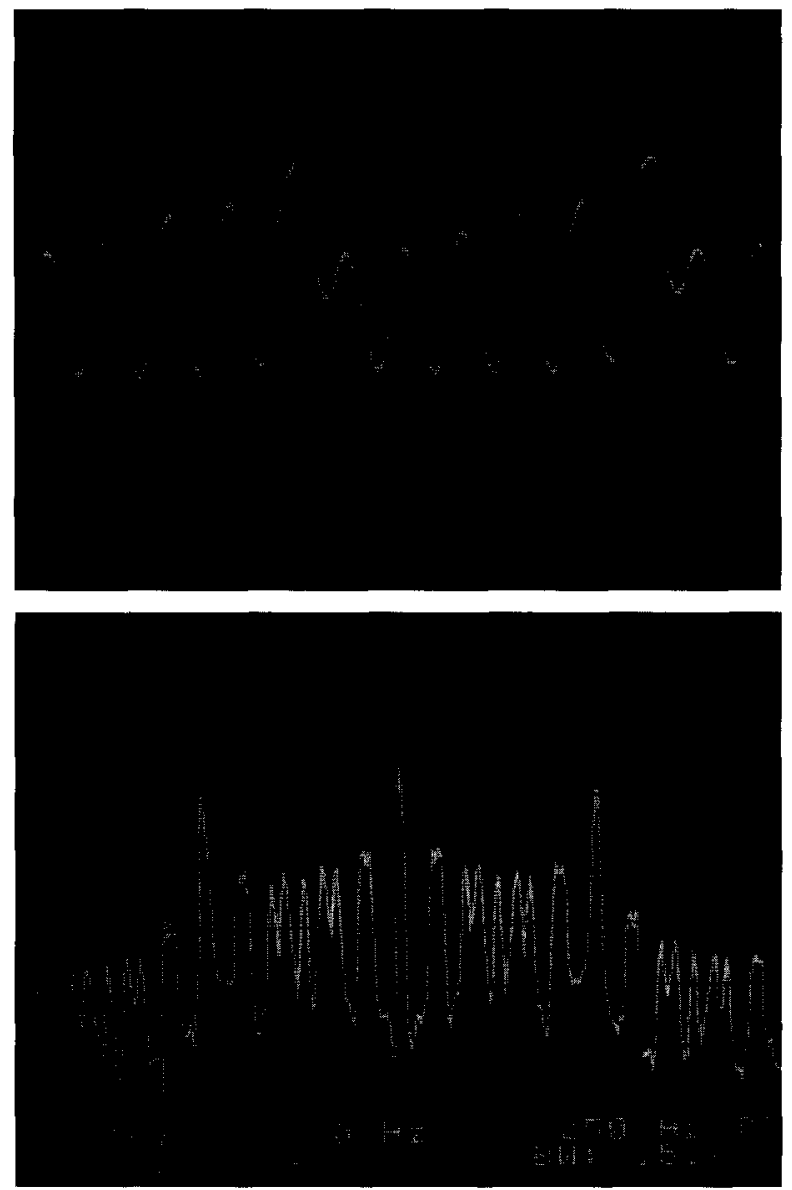

FIG. 9(b).

only the spectrum pattern without changing the fundamental frequency of the signal generated by the circuit. We refer to the fundamental frequency as the tenor of a signal to distinguish it from the perceived pitch of the signal (23). The perceived pitch may be lower than the tenor due to period-adding or period-doubling bifurcations.

\section{Application to Musical Signal Generation and Performance}

In this section we discuss methods for controlling the analog Chua's circuit for music composition and performance. In the general case, including both traditional and electronic music, musical signal generation can be described as a process of real-time application of control signals, including human gestures, to a complex system, such as a musical instrument consisting of multiple elements. To create musical signals we need fine-grain time specification of events, fine-grain increments for control signals, and fast multidimensional control of the signal-generating 

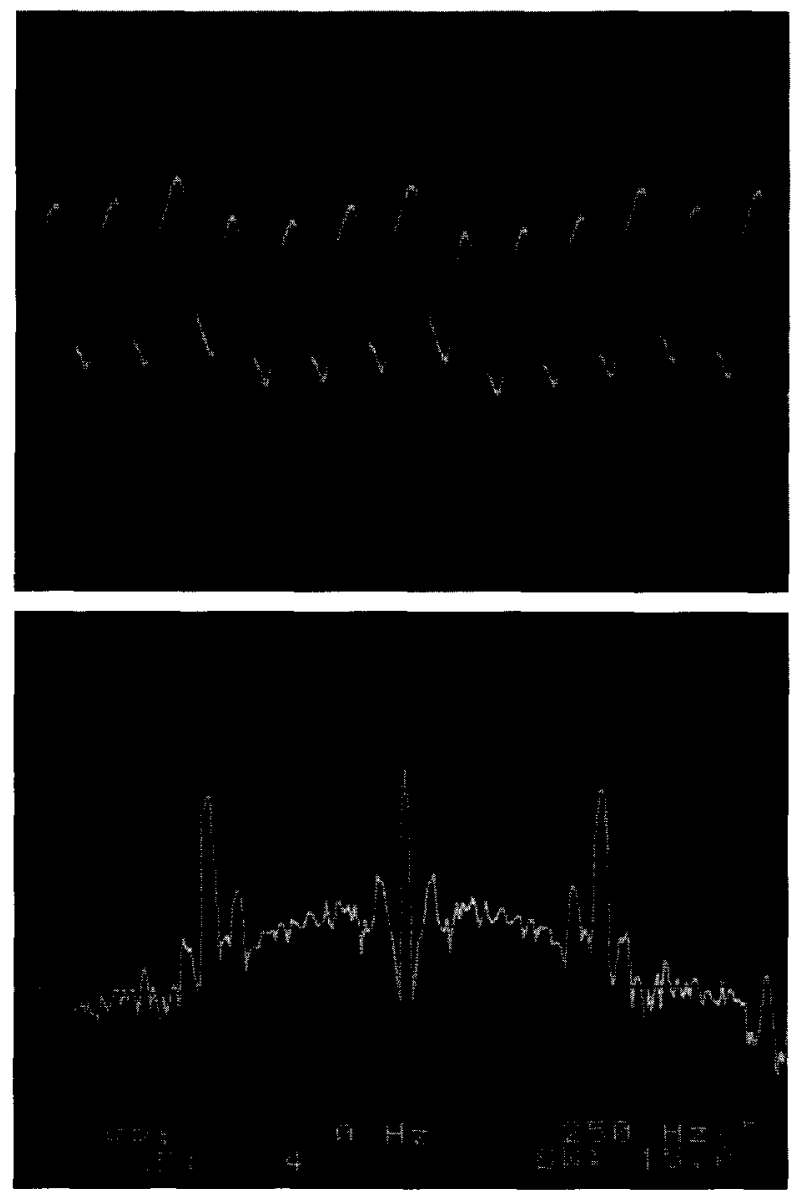

FIG. 9(c).

device. When tuning control parameters of musical signals, a parameter update rate faster than $100 \mathrm{~ms}$ is required to ensure the controls have their intended result in the auditory domain. Slower update rates may create audible discontinuities even when the control signals are varied smoothly and continuously. (Bifurcations create a different class of discontinuities which may be desired in auditory signals.) For the Chua's circuit we generate control signals from computer software to achieve rapid rates for updating control values, and for accurate reproduction of multiple simultaneous control signals.

\subsection{Explorations using an interactive digital simulation of Chua's circuit}

Initial investigations of musical properties were conducted with a digital simulation of the unfolded Chua's circuit (19) implemented in the $\mathrm{C}$ programming language with ordinary difference equations and Runge-Kutta integration. This simulation runs in a real-time sound synthesis environment $(\mathbf{2 4}, \mathbf{2 7})$ where the simulated voltages are converted to analog voltages at a $32 \mathrm{kHz}$ sampling rate, 

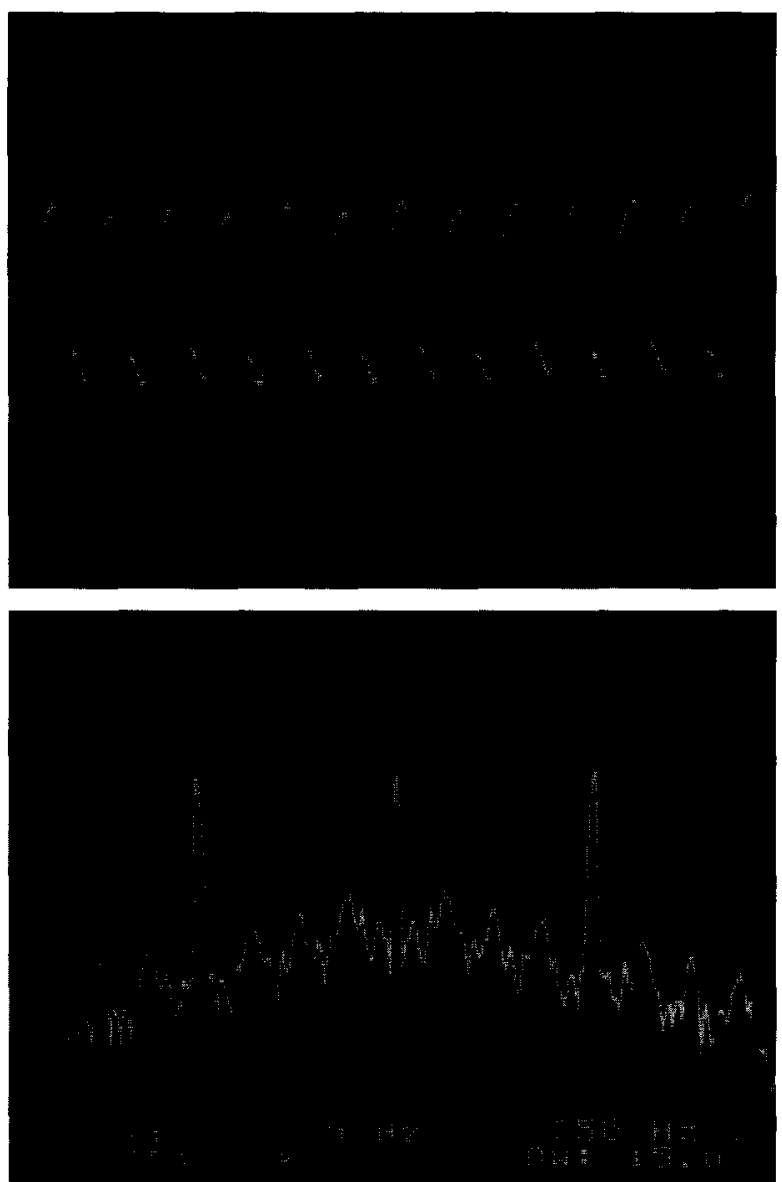

FIG. 9(d).

producing an auditory signal. A graphical interface adapted from Rodet (21) depicts a control fader for each circuit parameter (Fig. 10), allowing interactive investigation of the acoustic effects caused when the parameter values are changed. Minima and maxima for each fader can be entered from the keyboard. By specifying a minimum and a maximum we create a parameter range. With respect to the fixed length of a fader, a narrow parameter range produces fine-grained parametcr changes and a wide parameter range produces coarse parameter changes. Results obtained indicate that simultaneous tuning of multiple parameters increases the control capability to stabilize the circuit in certain regions of unstable periodic limit cycles, also to induce system migration from one basin of attraction to another, both of which are desirable for composing musical signals from chaotic signals.

\subsection{An analog circuit for real-time performance}

For real-time musical signal production a digital simulation is subjected to frequency range restrictions. The Nyquist frequency $(\mathrm{SR} / 2)$ determines the upper 

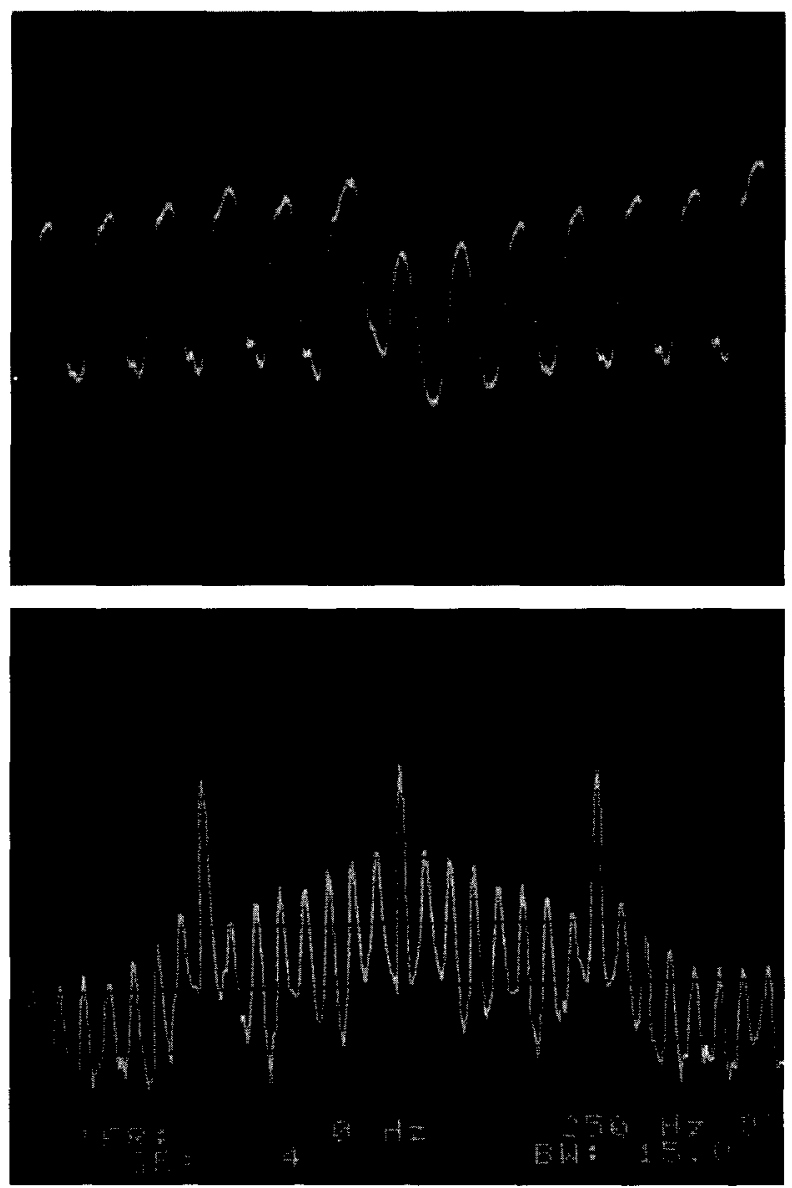

FIG. 9(e).

limit of frequencies a digital signal can reproduce. To cover the full range of human hearing (up to about $20 \mathrm{kHz}$ ) a circuit simulation must compute samples and convert them to analog voltages at rates exceeding $40 \mathrm{kHz}$. On many computers this computation is too expensive to be performed for real-time sound production. An experimental Chua's circuit avoids these computational restrictions, and can produce tones containing more complete spectral information than tones from a digital simulation. The high-frequency spectral components convey significant musical information concerning articulation, brightness, spectral centroid (a characteristic of timbre) and loudness (28).

To transform an electronic circuit into a musical signal generator we provide a method for fast interactive tuning of its parameters, and methods to attenuate, amplify, and propogate the signal into a listening space. For interactive tuning the seven voltage-controlled parameters of Chua's circuit are connected to a digital computer via a $\mathrm{D} / \mathrm{A}$ converter; for auditory display the circuit output was con- 


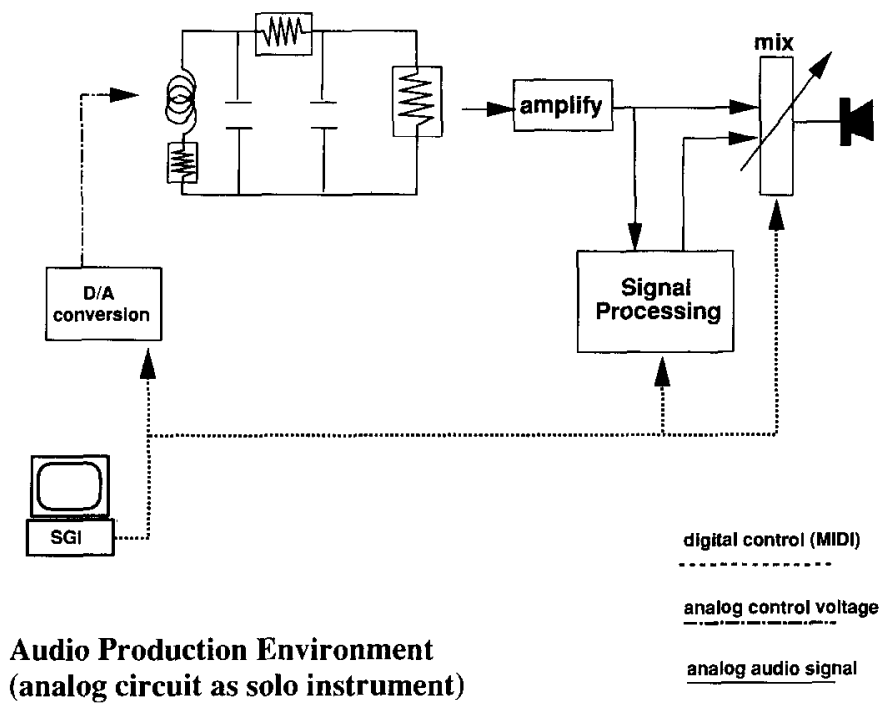

FIG. 10. The graphical interface depicting a control fader and a minimum and maximum value for each circuit parameter.

nected to an audio amplifier and reproduction system that could also be controlled from the computer (Fig. 11).

\subsection{Controlling multiple circuit parameters in real-time}

Programmable software provides independent simultaneous tuning of $n$ digital signals. These digital control signals are transmitted from the computer using the MIDI (Music Instrument Digital Interface) specification, a standard serial protocol developed by the electronic music instrument manufacturing industry $(29,30)$. Data is transmitted using a serial current-loop line and a $5 \mathrm{~mA}$ on/off signal in the order one start bit, eight data bits and one stop bit. Control signals are specified in integer values in the range $\{0, \ldots, 127\}$. Then $\mathrm{D} / \mathrm{A}$ conversion of this range into $\{0,10\}$ volts is accomplished with an off-the-shelf MIDI to CV converter (the MIDI Retro/XLV manufactured by Clarity corporation); further scaling of this voltage range was performed for each parameter (see below). The MIDI transmission rate of $31.25 \mathrm{~K}$ baud ensures the parameters are updated in rapid sequences which the ear detects as simultaneous.

To correctly tune the parameters of the Chua's circuit for musical tones, the $\{0$, $10\}$ voltage range of the Retro/XLV output is rescaled into a specific parameter range at each circuit component. Parameter ranges are collectivcly spccified such that the signal trajectory of the circuit does not converge onto the large limit cycle (LLC), a sine-like period-one signal corresponding to the outer breakpoints of the driving point characteristic of $N_{R}$. The LLC produces a steady pure tone that is musically uninteresting. Parameter ranges for more than one circuit component have an interdependent influence upon the signals produced. The parameter value of any one of the circuit components helps define the effects produced by traversing the parameter range of any other component. The selection of minimum and 


\section{Graphical Interface for controlling the Chua's circuit simulation}

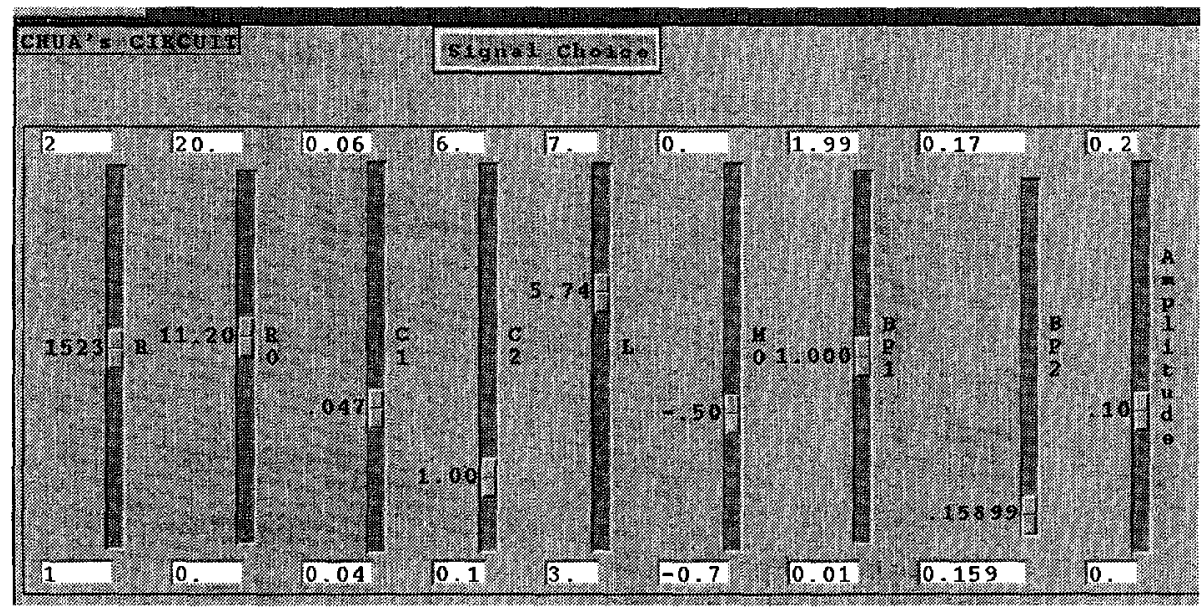

FIG. 11. The musical signal generation system for controlling the parameters of the Chua's circuit in real-time to produce specific audio signals, also for providing audio signals from a digital simulation of the Chua's circuit, and for controlling the signal processing and musical presentation of all of the audio signals.

maximum values for individual components is accomplished by experimentally increasing and decreasing a parameter's value while listening for desirable acoustic states and transitions. The specification of a parameter range for more than one circuit component defines a region of control space we refer to as a parameter region. A parameter region determines the acoustic neighborhood within which interactive gestures produce signals. Our goal has been to identify regions containing interesting ranges of sounds, and find the simplest combination of parameter changes to arrive at those sounds.

The specification of a parameter range for each component of the analog circuit is a design concept descended from tests with the digital simulation (see upper and lower value windows for each fader in Fig. 10). To implement range specifications in an experimental circuit, each component of the multiple-control circuit is attached to a control signal scaling component consisting of an op amp and two voltage-controlled resistors. Each pair of resistors is used to specify a minimum and maximum voltage by which the $\{0,10\} v$ Retro/XLV output signal is scaled (31). In the performance implementation of the circuit, scaling is manually adjusted based upon a set of fiducial points that are audible and also may be viewed on an oscilloscope. Stable periodic limit cycles provide good fiducial points due to their easily-observed phase, their nearly-pure harmonic content and their unambiguous pitch.

\subsection{Control signal discretization}

Acoustic continuity and circuit signal stability depend in part on fine-grained $\Delta v$ and $\Delta t$. For the analog circuit $\Delta t$ is determined by the speed of the resistors and is 
typically $10 \mu \mathrm{s}(\mathbf{2 2})$. The value of $\Delta v$ is determined by the amplitude resolution of the digital control signal. The analog circuit has been controlled by a relatively coarse-grained 7-bit MIDI signal. By contrast, the digital simulation may be controlled by digital signals in double-precision floating point resolution. We have observed a discrepency in circuit output signals using these different resolutions of control signal. With a MIDI-controlled analog circuit we have observed that a large parameter range can result in a low-resolution control signal which may have two effects differing from the simulation, namely (1) producing audible discontinuities during a transition between circuit states whereas the same transition is acoustically continuous using the simulation, and (2) reducing our capability to entrain the signal in certain basins of attraction. During the course of experiments and again during the course of music composition, we were able to produce states in the digital simulation that could not be reproduced in the analog circuit. This discrepency is easily noticed when searching for a memorable sound from the analog circuit, which has already been produced in the digital simulation. This experience leads us to conclude that the MIDI protocol may not provide sufficiently fine-grain control for musical applications.

\section{Bifurcation Sequences and Attractors}

In this section we present a bifurcation sequence and attractors as a function of the voltage-controlled $R$. We also present some samples of bifurcation sequences and attractors as a function of the voltage-controlled $L$, breakpoints $+E$ and $-E$, as well as slopes $G_{a}$ and $G_{b}$ of the piecewise-linear resistor $N_{R}$ 's $v-i$ characteristic.

\subsection{Bifurcation sequence with respect to $R\left(v_{c}\right)$}

By increasing the controlling voltage $v_{c}$ from $+4.181 \mathrm{~V}$ towards $+5.519 \mathrm{~V}$, i.e. reducing $R\left(v_{c}\right)$ from $1.638 \mathrm{k} \Omega$ towards $1.253 \mathrm{k} \Omega$, Chua's circuit exhibits a sequence of bifurcation from a de equilibrium point through a Hopf bifurcation and perioddoubling sequence to a spiral Chua's attractor and the double scroll Chua's attractor, and finally a large limit cycle, as shown in Fig. 12(a)-(m). Some oscilloscope pictures of the time-waveforms and spectra are presented in these figures, too. Note that a rich variety of spectra patterns are observed.

\subsection{Bifurcation sequence with respect to $L$}

A bifurcation sequence similar to that of changing $R$, can be observed from Chua's circuit by adjusting the controlling voltage for $L\left(v_{c}\right)$. Here we show only two attractors, and samples of accompanying waveforms and spectra, as shown in Fig. 13(a)-(b). Note from the spectrum diagrams in the figures that the fundamental frequency is changed as the value of the parameter $L$ is varied.

\subsection{Bifurcation sequence with respect to slopes}

The slopes $G_{a}$ and $G_{b}$ of $N_{R}$ 's $v-i$ characteristic will affect the shape of the attractor, and consequently the spectrum pattern. The range of the slopes, however, is rather narrow when other parameter values of the circuit are fixed. We present 


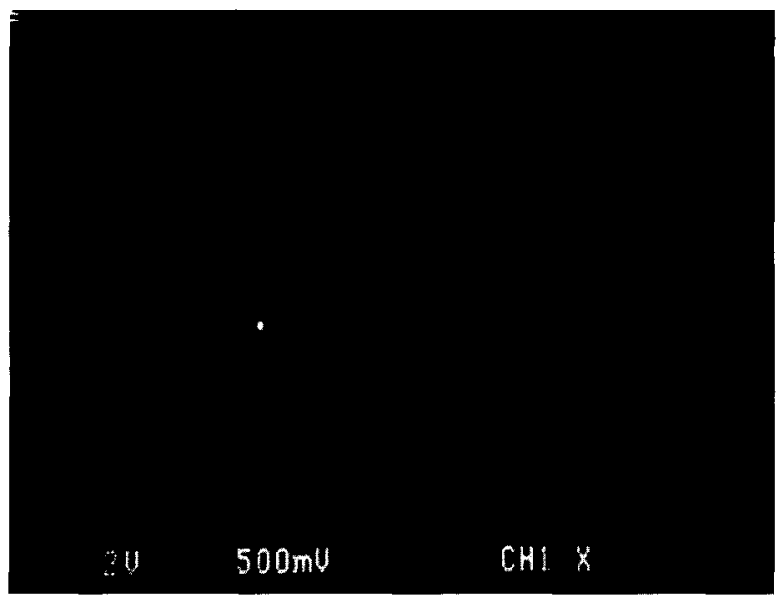

FIG. 12(a). Bifurcation sequence with respect to $R\left(v_{c}\right)$.

Horizontal axis $v_{c 1}$, scale: (a)-(d) $2 \mathrm{~V} /$ div, (e)-(l) $1 \mathrm{~V} /$ div, (m) $2 \mathrm{~V} /$ div, for trajectories; Horizontal axis $t$, scale: (b) $1 \mathrm{msec} / \mathrm{div}$, (c)-(d) $2 \mathrm{msec} / \mathrm{div}$, (e)-(h) $5 \mathrm{msec} / \mathrm{div}$, (i)-(k) 2 $\mathrm{msec} / \mathrm{div}$, for time waveforms; Horizontal axis $f$, scale: (b)-(k) $250 \mathrm{~Hz} / \mathrm{div}$, for spectra; Vertical axis $v_{c 2}$, scale : (a)-(d) $500 \mathrm{mV} /$ div, (e)-(l) $200 \mathrm{mV} /$ div, (m) $1 \mathrm{~V} /$ div, for trajectories; Vertical axis $v_{c 1}$, scale : (b)-(e) $2 \mathrm{~V} / \mathrm{div}$, (f)-(k) $1 \mathrm{~V} / \mathrm{div}$, for time waveforms; Vertical axis $v_{c 1}$, scale : $10 \mathrm{~dB} /$ div, for spectra.

Parameter values: $C_{1}\left(v_{c}\right)=24.45 \mathrm{nF}, C_{2}\left(v_{c}\right)=368.6 \mathrm{nF}, L\left(v_{c}\right)=38.08 \mathrm{mH}$ (with inherent series resistance $28.65 \Omega$ ), $R_{0}\left(v_{v}\right)=6.32 \Omega, G_{a}=-0.72 \mathrm{mS}, G_{b}=-0.5 \mathrm{mS}, \pm E= \pm 1 \mathrm{~V}$.

(a) $v_{c}=1.797 \mathrm{~V}, R\left(v_{c}\right)=1.638 \mathrm{k} \Omega$, dc equilibrium point.

two attractors for the examples here as the slopes are controlled, as shown in Fig. 14(a)-(b).

\subsection{Bifurcation sequence with respect to breakpoints}

The locations of the breakpoints of the $N_{R}$ 's $v-i$ characteristic will also affect the shape, and consequently the spectrum pattern of the attractor. Moving the breakpoint closer to the origin will shrink or even suppress one of the lobes in the double scroll Chua's attractor. As an example, we show four attractors shrunk by moving $+E$ and $-E$ closer to the origin, respectively, as shown in Fig. 15(a)-(d). Note from the pictures that different spectra patterns are obtained as the breakpoints move.

\section{Concluding Remarks}

Chua's circuit has become a paradigm for studying chaotic dynamics since the circuit was invented by Chua 10 years ago. The circuit can exhibit a rich variety of bifurcation sequences and attractors as one or more parameter values of the circuit are changed. Chaotic circuits such as this produce both harmonic and noiselike (unstable) signal characteristics, which are important components in musical signals. The analog Chua's circuit is an efficient source of complex signals, and the 

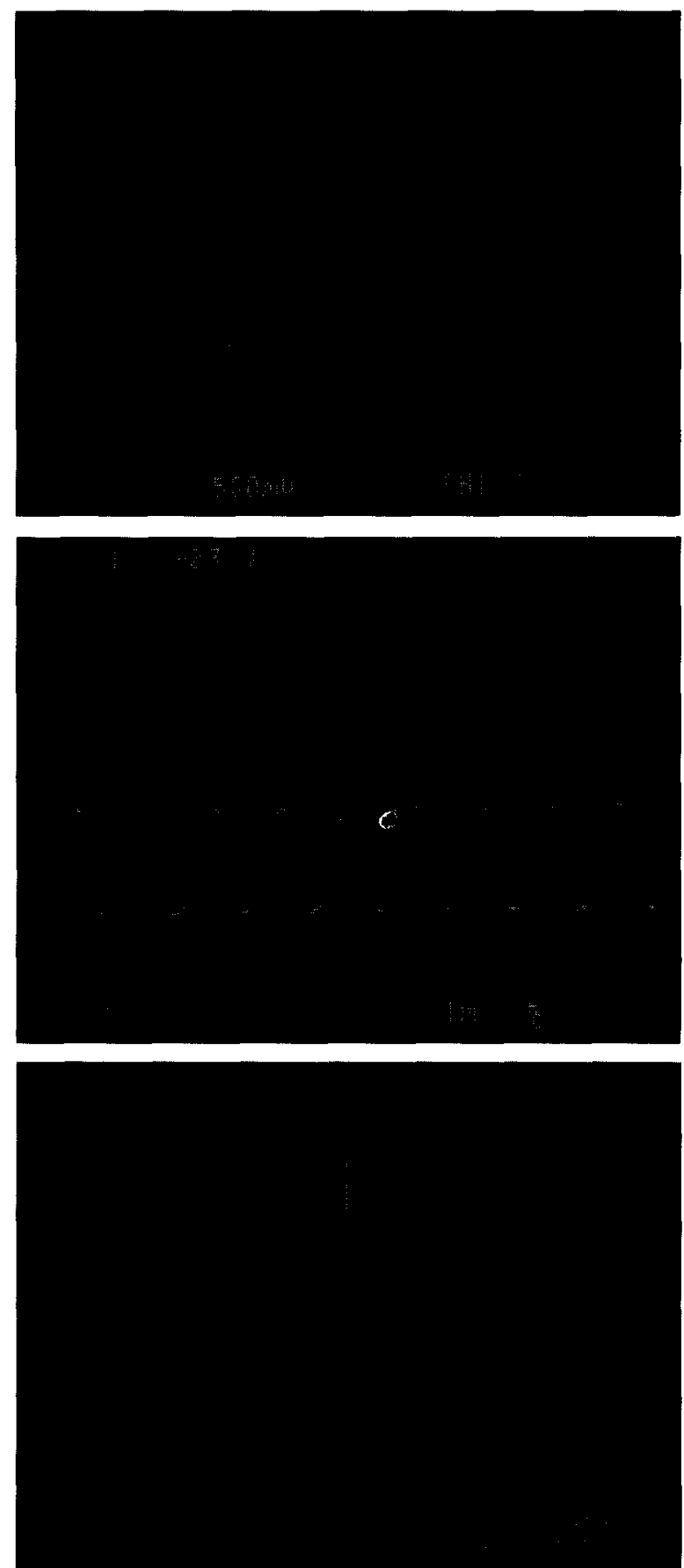

FIG. 12(b) $v_{c}=1.922 \mathrm{~V}, R\left(v_{c}\right)=1.591 \mathrm{k} \Omega$, period-1 
G. - Q. Zhong et al.
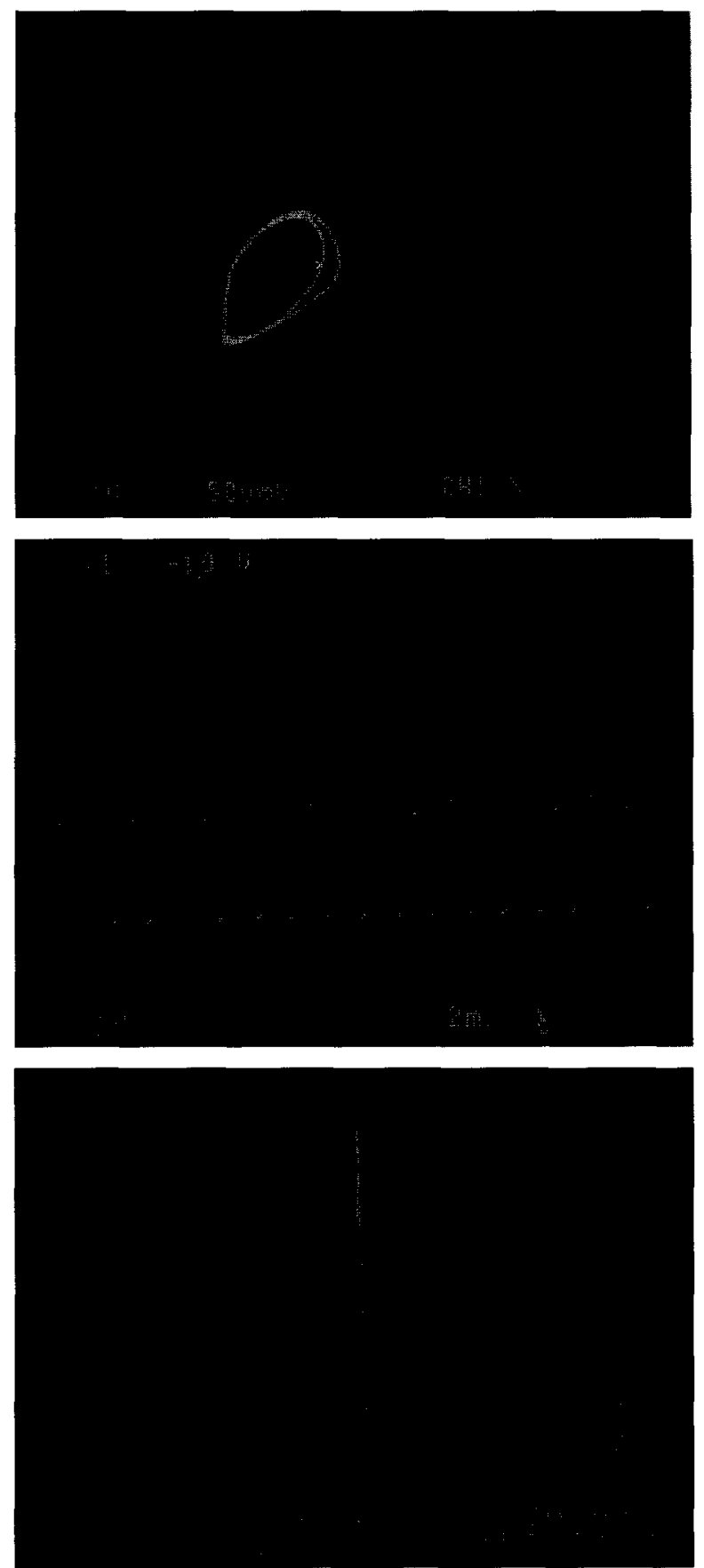

FIG. $12(\mathrm{c}) v_{c}=1.952 \mathrm{~V}, R\left(v_{c}\right)=1.581 \mathrm{k} \Omega$, period-2. 

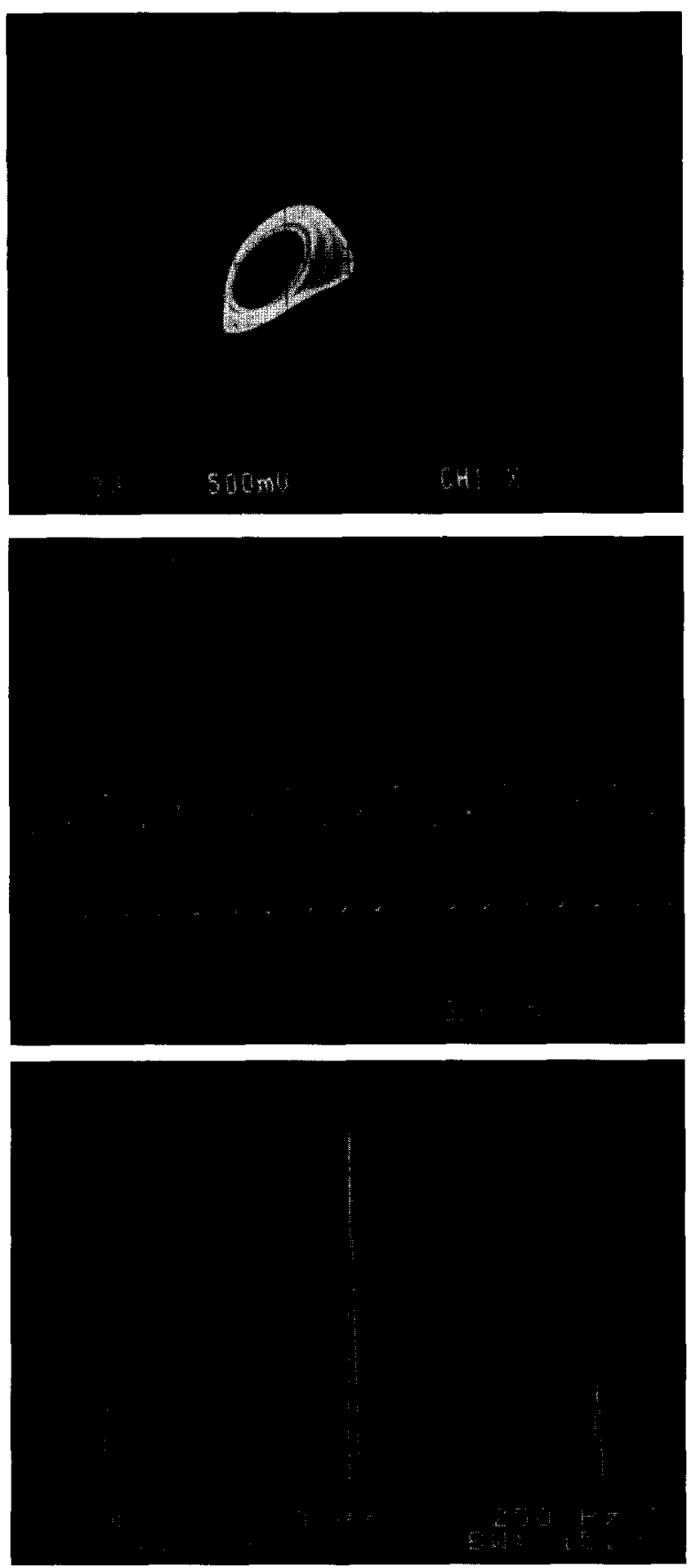

FIG. $12(\mathrm{~d}) v_{c}=2.002 \mathrm{~V}, R\left(v_{c}\right)=1.569 \mathrm{k} \Omega$, Rössler-type attractor. 
G.-Q. Zhong et al.
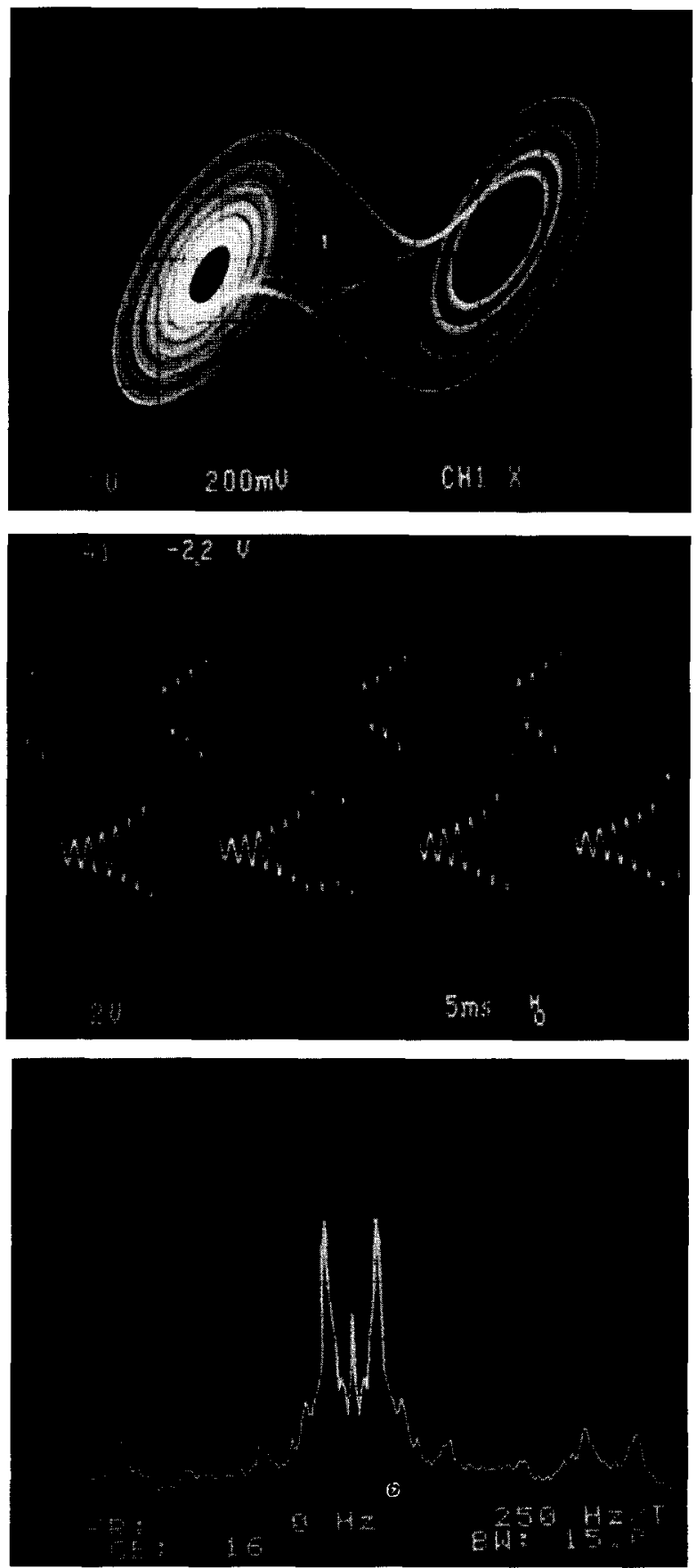

FIG. $12(\mathrm{e}) v_{r}=2.178 \mathrm{~V}, R\left(v_{c}\right)=1.533 \mathrm{k} \Omega$, double scroll attractor. 

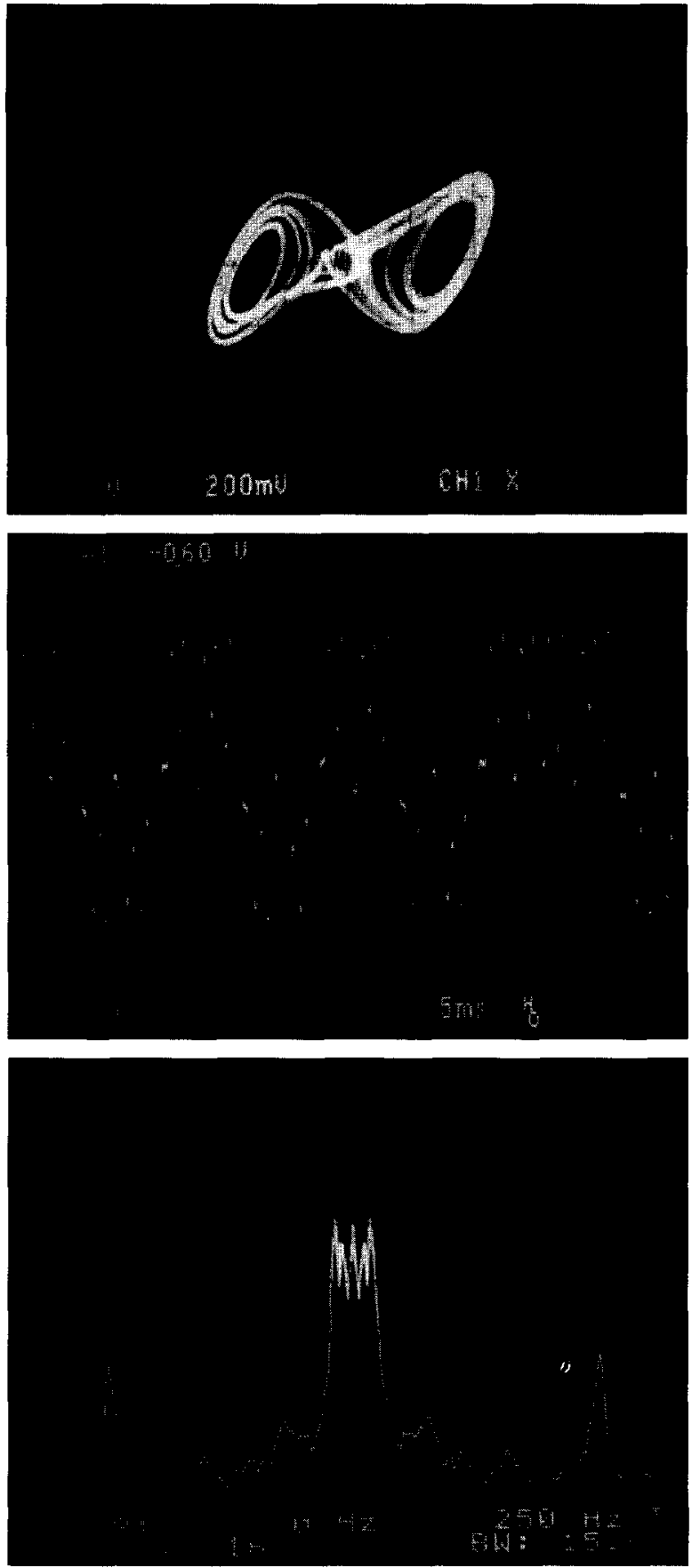

FIG. $12(\mathrm{f}) v_{c}=2.746 \mathrm{~V}, R\left(v_{c}\right)=1.433 \mathrm{k} \Omega$, double scroll attractor. 
G.-Q. Zhong et al.
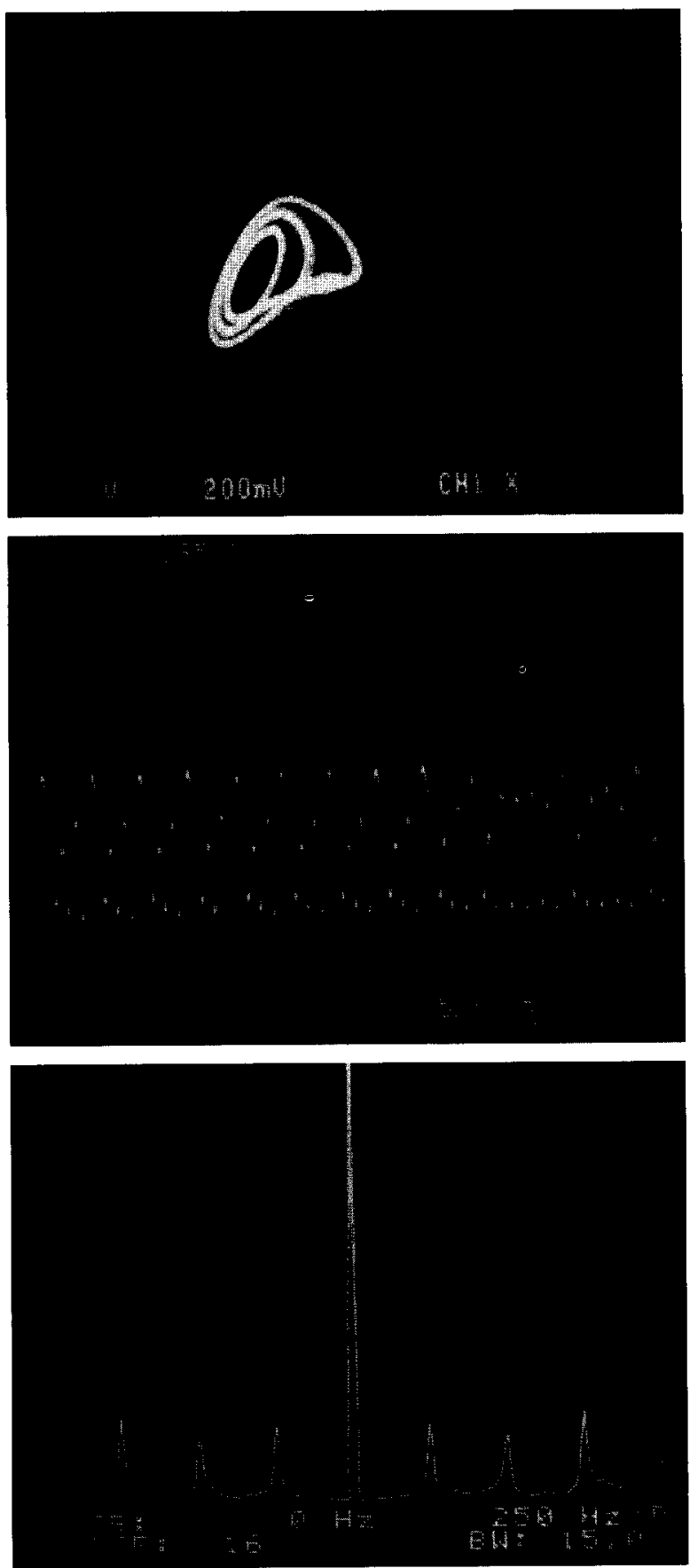

FIG. $12(\mathrm{~g}) v_{c}=2.778 \mathrm{~V}, R\left(v_{c}\right)=1.427 \mathrm{k} \Omega$, Rössler-type attractor. 

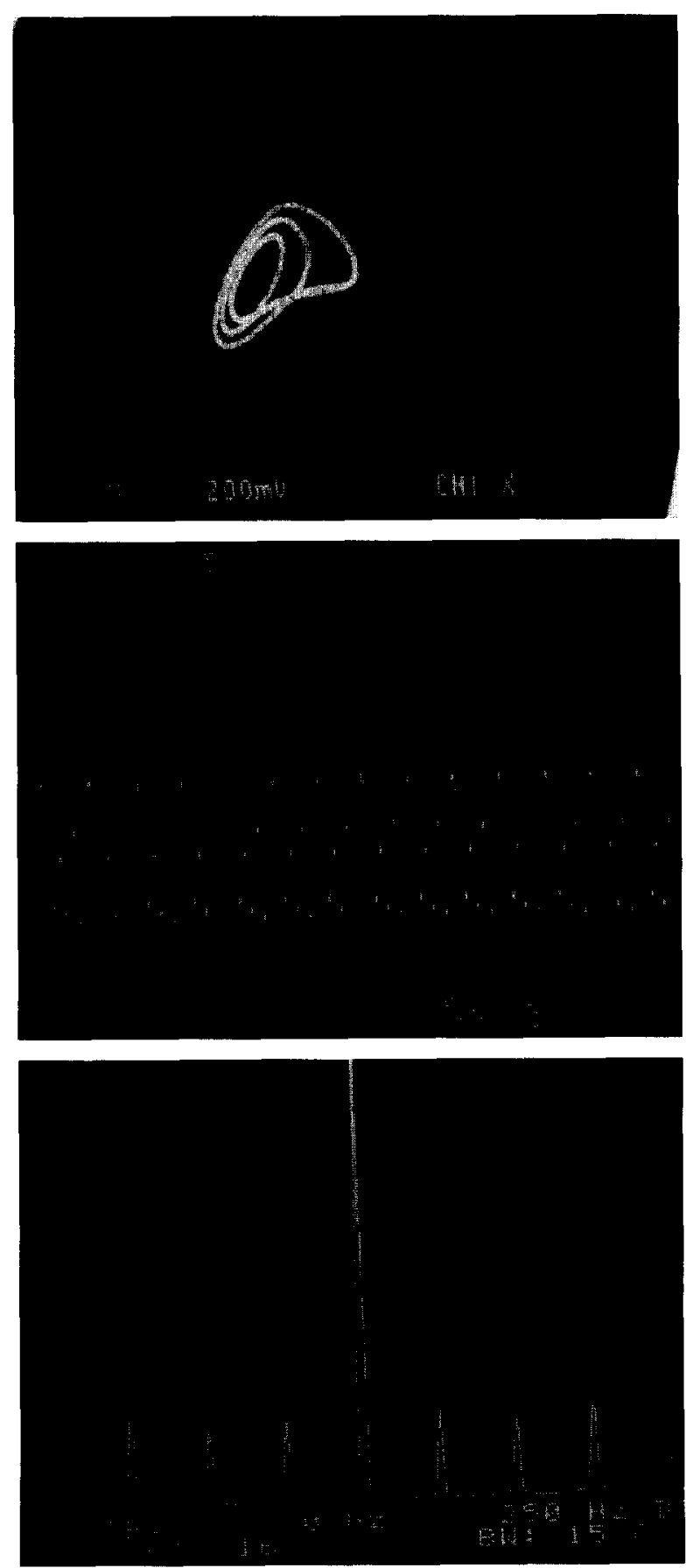

FIG. $12(\mathrm{~h}) v_{c}=2.799 \mathrm{~V}, R\left(v_{c}\right)=1.424 \mathrm{k} \Omega$, period-3 window. 
G.-Q. Zhong et al.
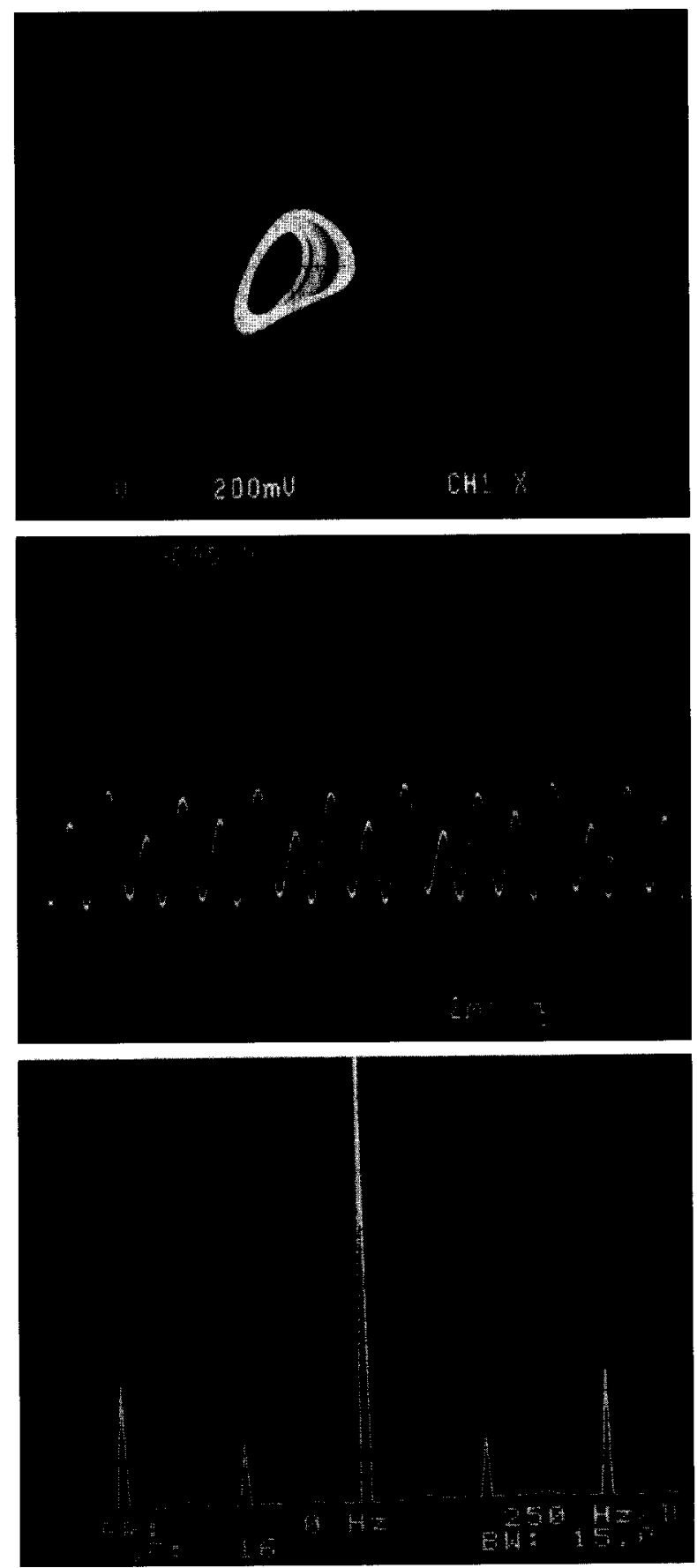

Fic. 12(i) $v_{c}=2.903 \mathrm{~V}, R\left(v_{c}\right)=1.404 \mathrm{k} \Omega$, Rössler-type attractor. 

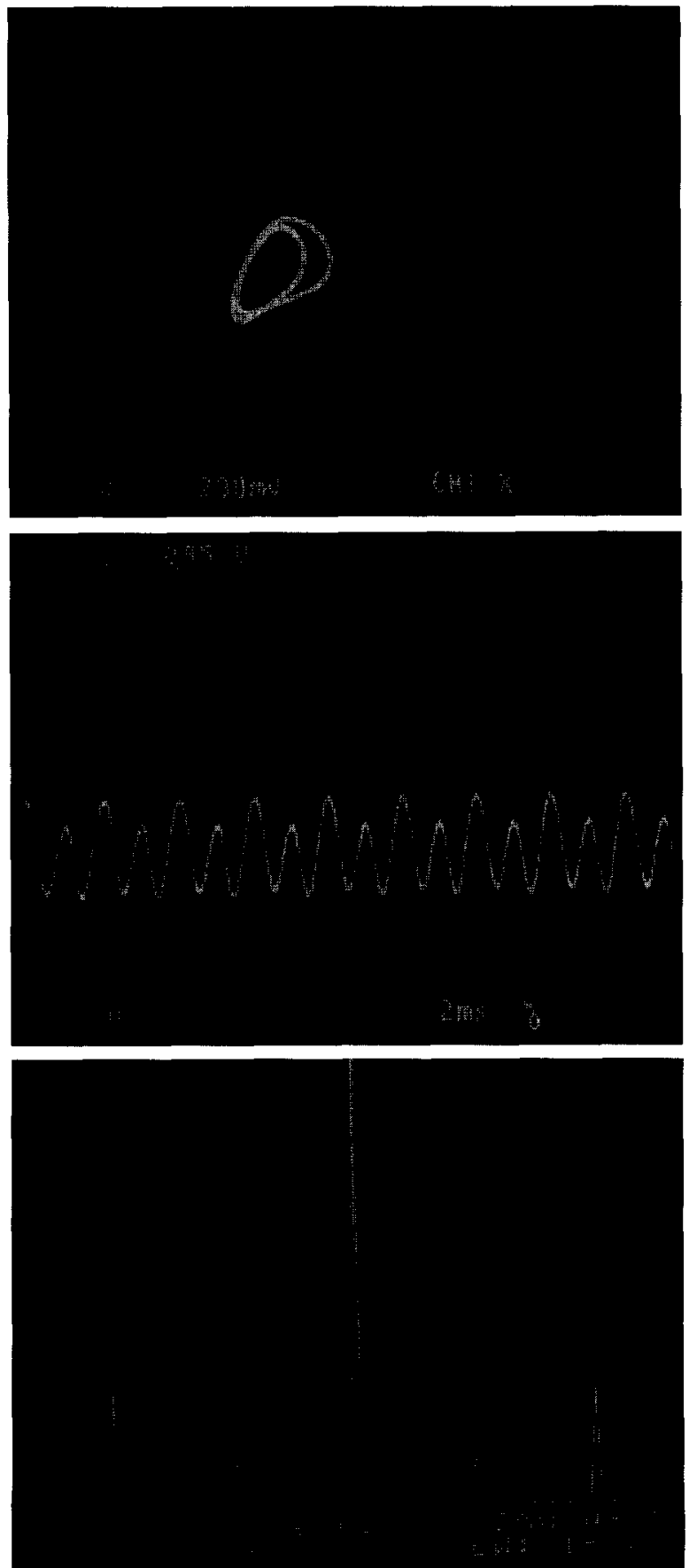

FIG. $12(\mathrm{j}) \boldsymbol{v}_{c}=2.975 \mathrm{~V}, \boldsymbol{R}\left(v_{c}\right)=1.388 \mathrm{k} \Omega$, period -2 . 
G.-Q. Zhong et al.
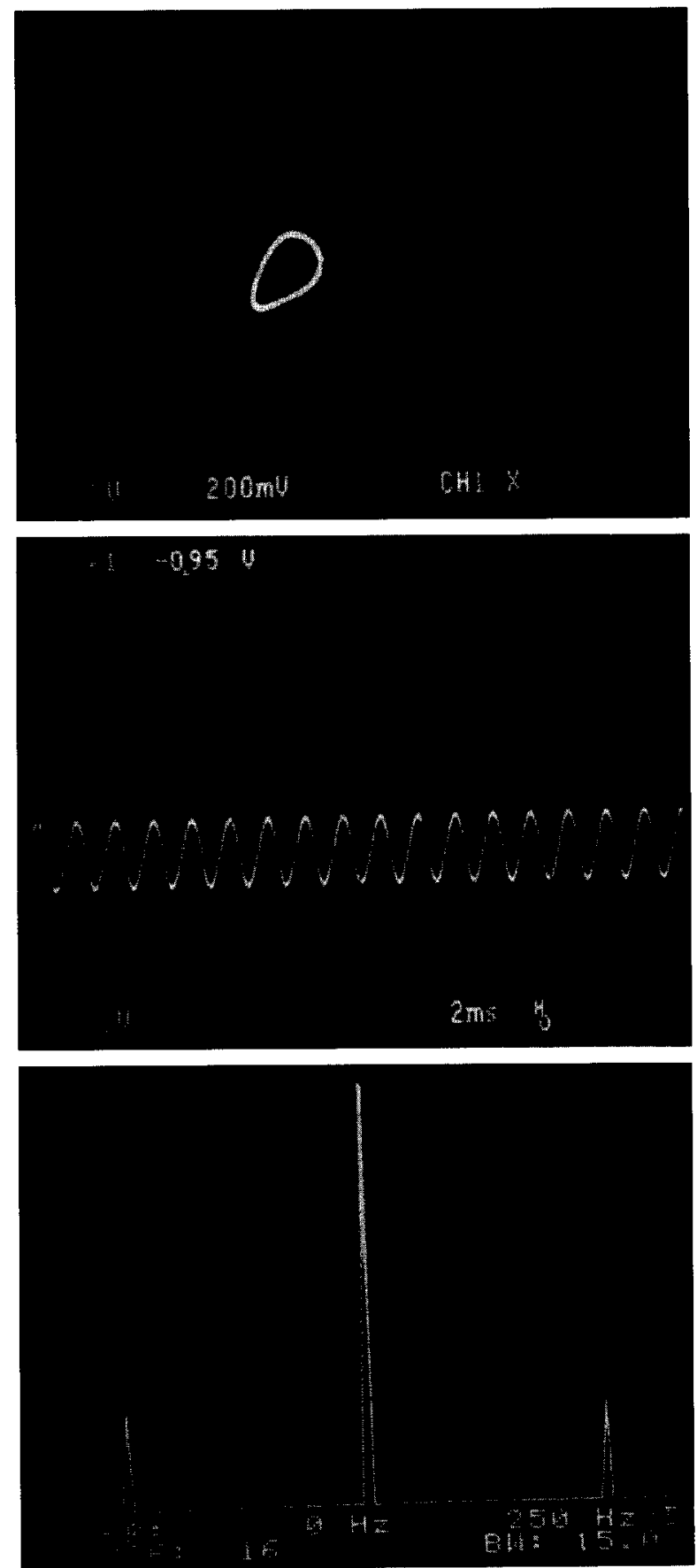

FIG. $12(\mathrm{k}) v_{c}=3.087 \mathrm{~V}, R\left(v_{c}\right)=1.363 \mathrm{k} \Omega$, period-1. 


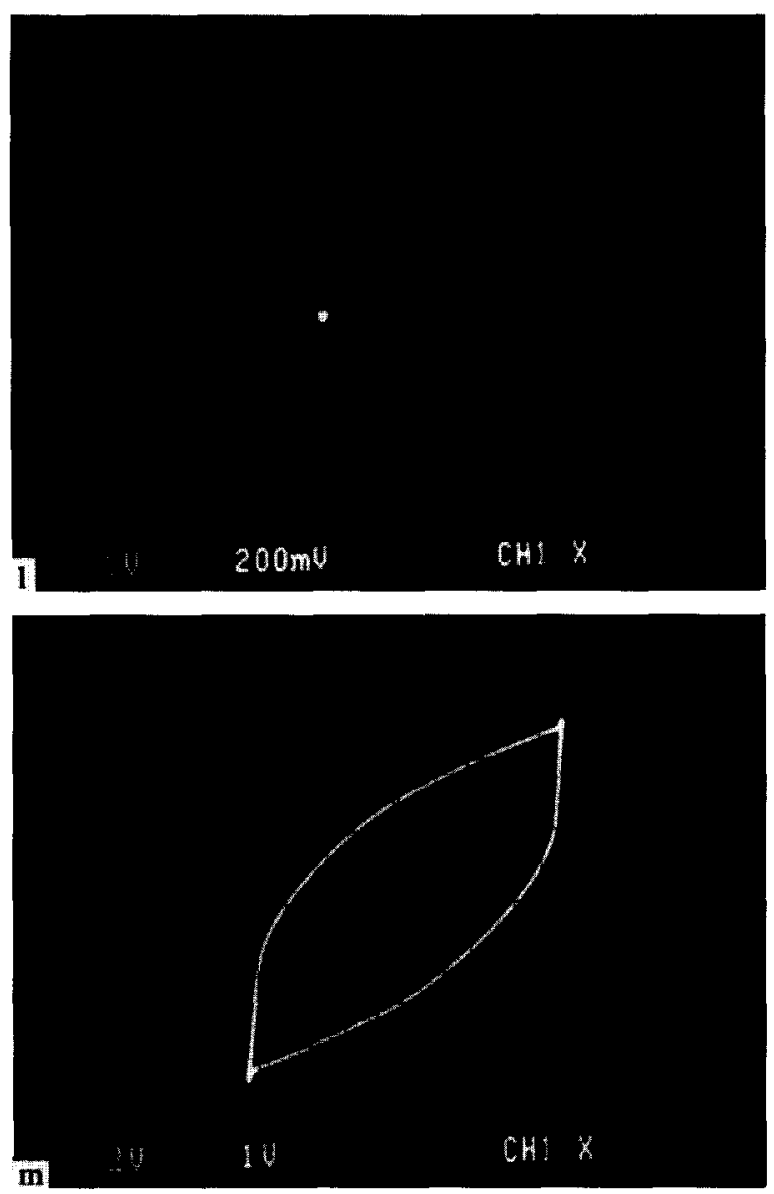

FIG. $12(1) v_{c}=3.317 \mathrm{~V}, R\left(v_{c}\right)=1.312 \mathrm{k} \Omega$, dc equilibrium point $;(\mathrm{m}) v_{c}=3.553 \mathrm{~V}, R\left(v_{c}\right)=$ $1.253 \mathrm{k} \Omega$, large limit cycle corresponding to outer segments of the $v-i$ characteristic.

simultaneous tuning of multiple parameters increases its efficiency. This paper presents a multiple-controlled Chua's circuit in which each parameter value of Chua's circuit can be voltage-controlled in real-time, regardless of the type of control signal, time-delayed or real-time. In this way, distinct bifurcation sequences, attractors, and spectrum patterns can be observed in real-time. The basic circuit discussed here has been utilized to successfully generate musical signals in live interactive computer-controlled performances of the composition "anti-Odysseus : the irreversibility of time" during Expo '93 in Seoul and Taejon, Korea (32).

Note from the observations presented in the paper that the fundamental frequency of the attractor will be changed when the energy-storage elements (capacitor and inductor) are adjusted. Adjustments of the other parameters in the circuit will only affect the spectrum patterns, i.e. the frequency components of the attractors. This property will benefit musicians when they utilize the multiple-control Chua's 

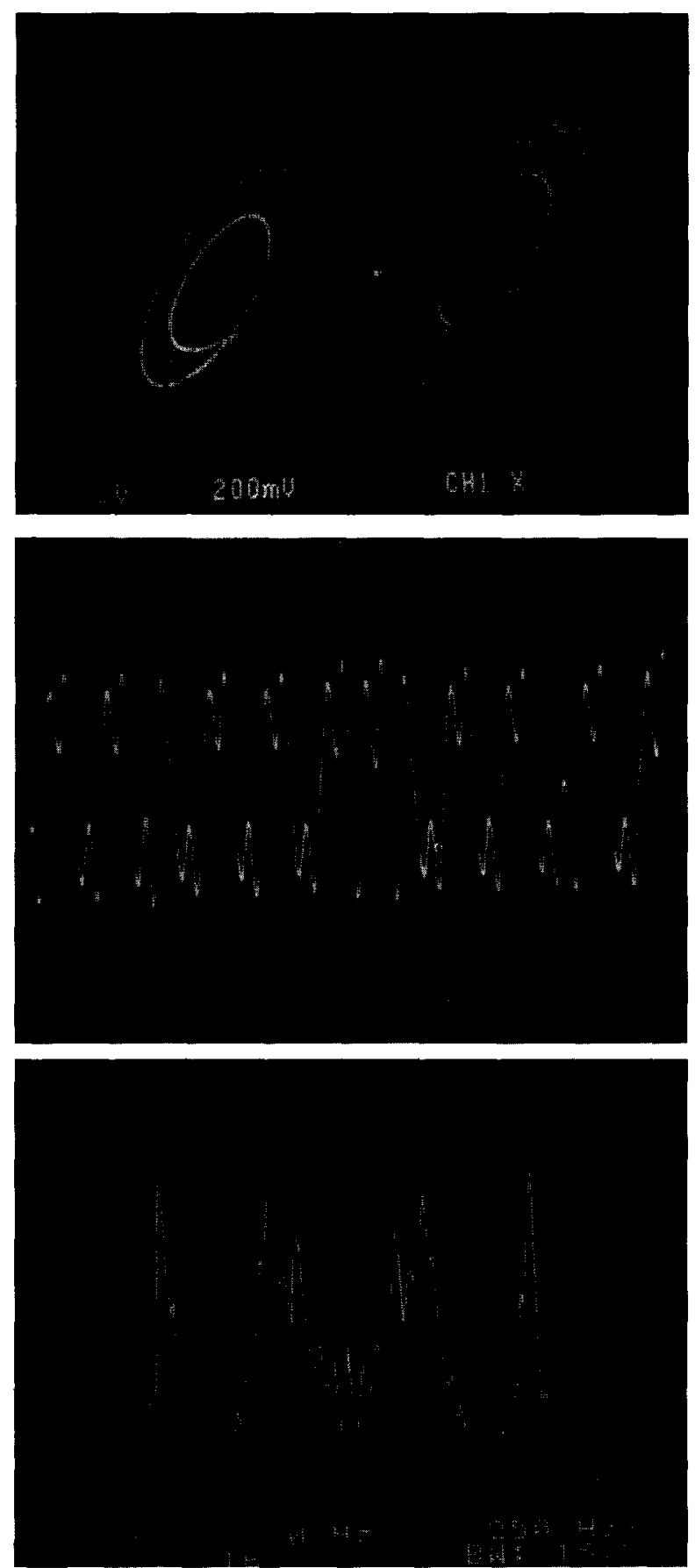

FIG. 13. Bifurcation sequence with respect to $L\left(v_{r}\right)$.

Horizontal axis $v_{c l}$, scale: $1 \mathrm{~V} / \mathrm{div}$, for trajectories; Horizontal axis $t$, scale: $5 \mathrm{~ms} / \mathrm{div}$, for time waveforms; Horizontal axis $f$, scale: $250 \mathrm{~Hz} /$ div, for spectra; Vertical axis $v_{c_{2}}$, scale: $200 \mathrm{mV} /$ div, for trajectories; Vertical axis $v_{c 1}$, scale : $2 \mathrm{~V} /$ div, for time waveforms; Vertical axis $v_{\mathrm{t}, 1}$, scale: $10 \mathrm{~dB} / \mathrm{div}$, for spectra.

Parameter values: $C_{1}\left(v_{c}\right)=24.45 \mathrm{nF}, C_{2}\left(v_{c}\right)=368.6 \mathrm{nF}, R\left(v_{c}\right)=1.533 \mathrm{k} \Omega, R_{0}\left(v_{c}\right)=$ $6.32 \Omega, G_{a}=-0.72 \mathrm{mS}, G_{b}=-0.5 \mathrm{mS}, \pm E= \pm 1 \mathrm{~V}$.

(a) $v_{c}=1.371 \mathrm{~V}, L\left(v_{c}\right)=44.11 \mathrm{mH}$ (with inherent series resistance $33.69 \Omega$ ). 

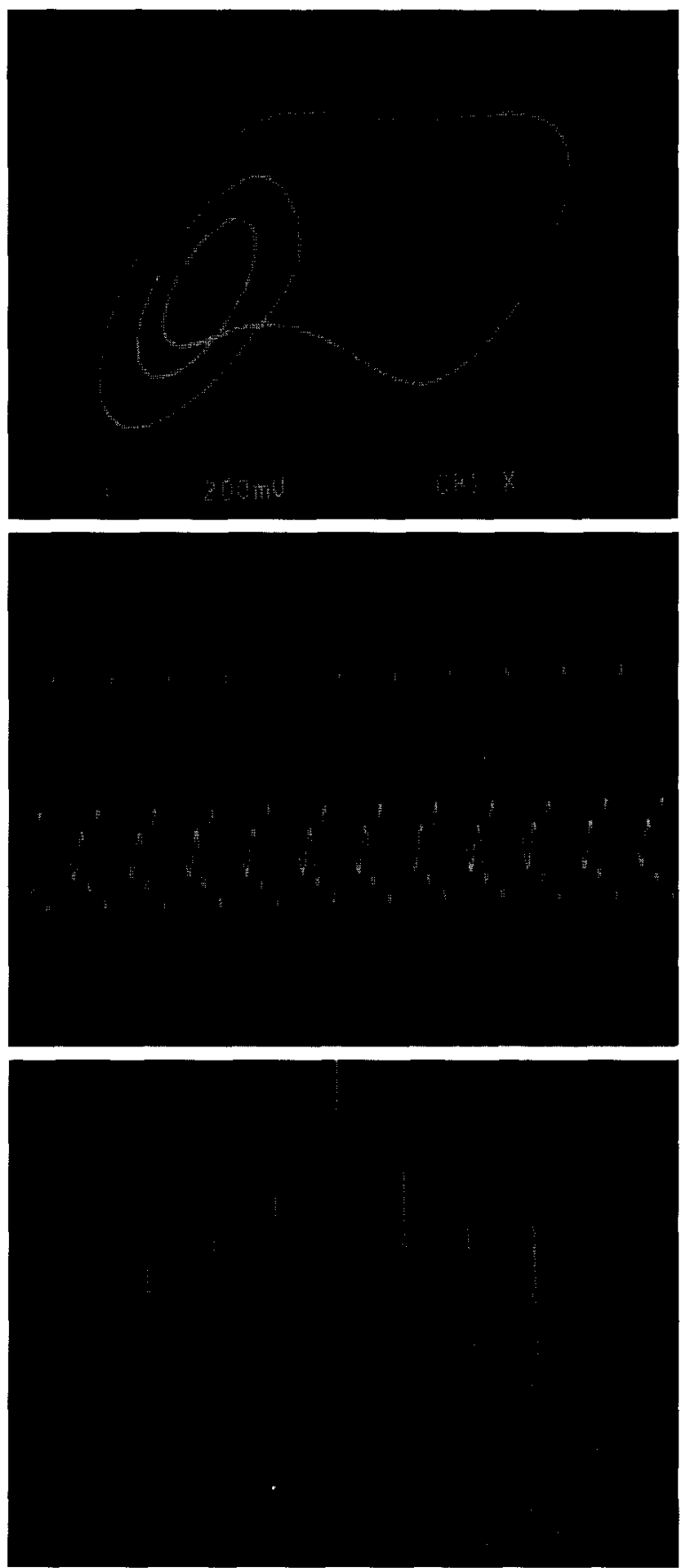

FIG. 13(b) $v_{c}=1.271 \mathrm{~V}, L\left(v_{c}\right)=43.62 \mathrm{mH}$ (with inherent series resistance $33.33 \Omega$ ). 

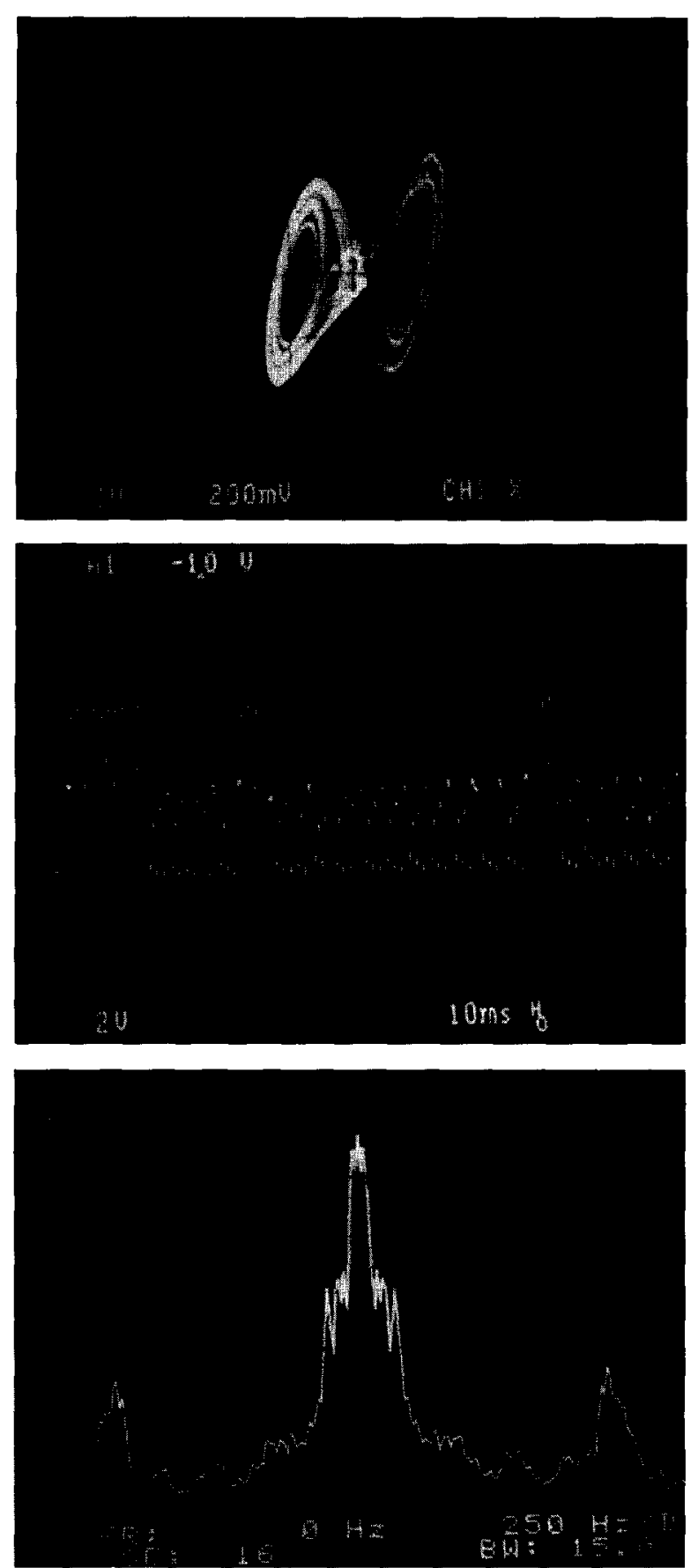

FIG. 14. Bifurcation sequence with respect to slopes.

Horizontal axis $v_{c l}$, scale: $2 \mathrm{~V} /$ div, for trajectories; Horizontal axis $t$, scale : (a) $10 \mathrm{~ms} / \mathrm{div}$, (b) $5 \mathrm{~ms} / \mathrm{div}$, for time waveforms; Horizontal axis $f$, scale : $250 \mathrm{~Hz} / \mathrm{div}$, for spectra ; Vertical axis $v_{c 2}$, scale: $200 \mathrm{mV} /$ div, for trajectories; Vertical axis $v_{r 1}$, scale: $2 \mathrm{~V} /$ div, for time waveforms; Vertical axis $v_{\mathrm{c}}$, scale: $10 \mathrm{~dB} / \mathrm{div}$, for spectra.

Parameter values: $C_{1}\left(v_{c}\right)=24.45 \mathrm{nF}, C_{2}\left(v_{c}\right)=368.6 \mathrm{nF}, R\left(v_{c}\right)=1.533 \mathrm{k} \Omega, R_{0}\left(v_{c}\right)=$ $6.32 \Omega, L\left(v_{r}\right)=38.08 \mathrm{mH}$ (with inherent series resistance $28.65 \Omega$ ), $\pm E= \pm 1 \mathrm{~V}$. (a) $v_{c}=$ $5.856 \mathrm{~V}, G_{a}=-0.7 \mathrm{mS}, G_{b}=-0.45 \mathrm{mS}$. 

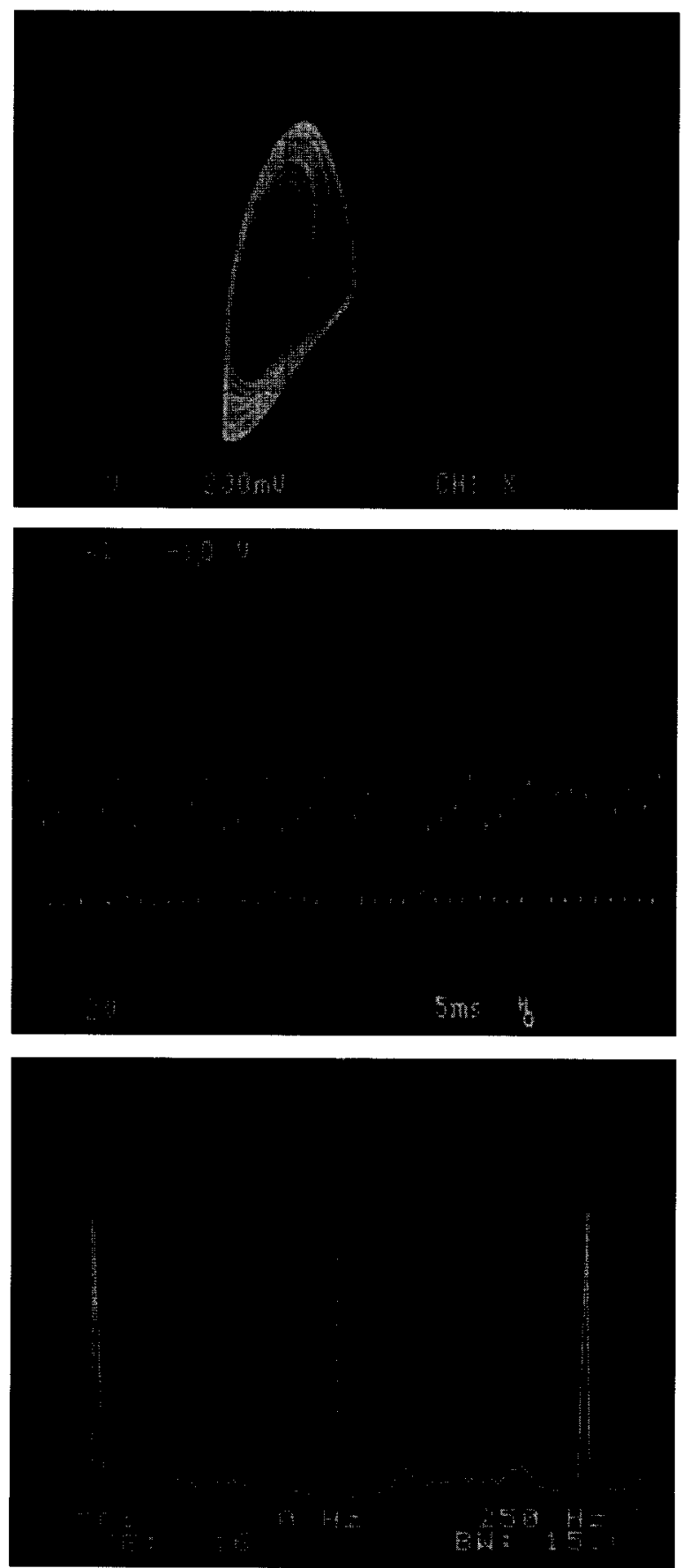

FiG. 14(b) $v_{c}=5.555 \mathrm{~V}, G_{a}=-0.77 \mathrm{mS}, G_{b}=-0.51 \mathrm{mS}$. 

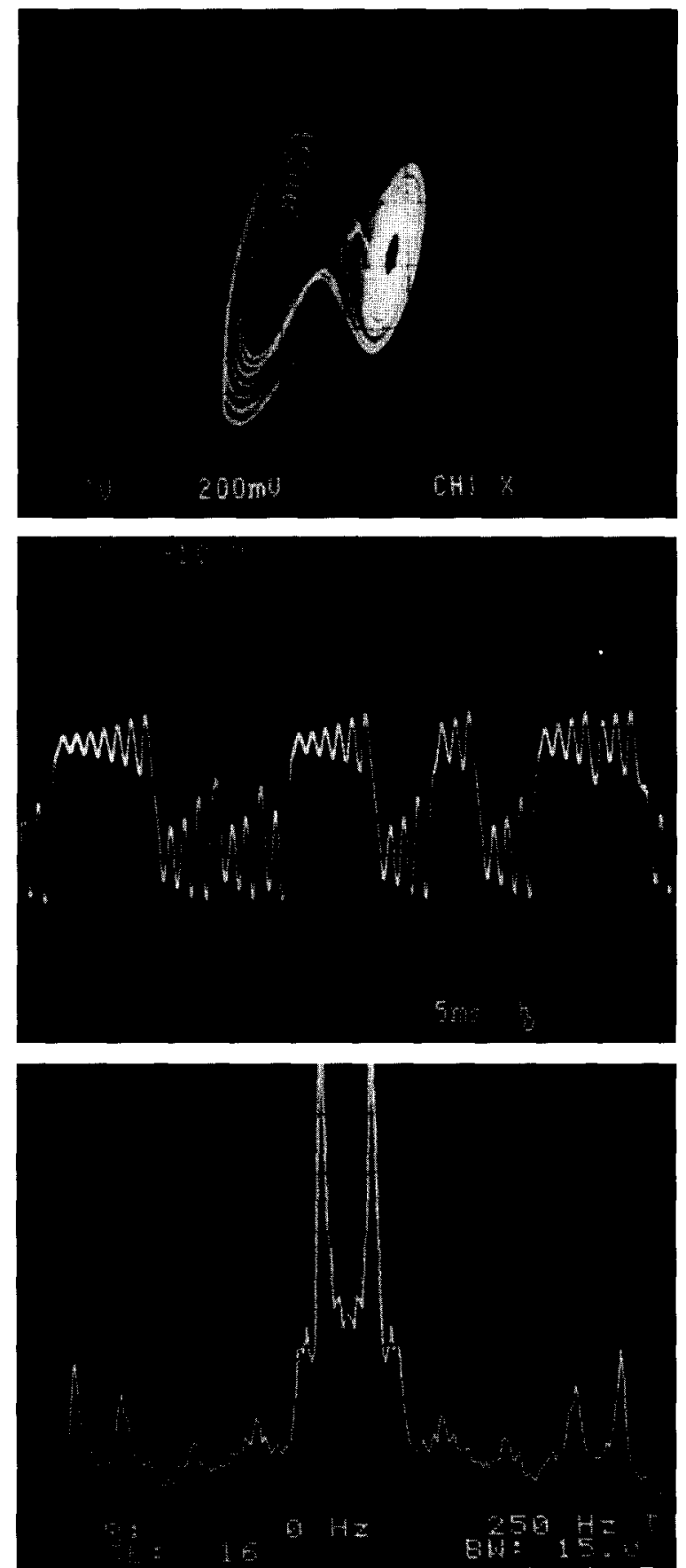

FIG. 15. Bifurcation sequence with respect to breakpoints.

Horizontal axis $v_{c 1}$, scale: (a), (c), (d) $2 \mathrm{~V} / \mathrm{div}$, (b) $1 \mathrm{~V} /$ div, for trajectories; Horizontal axis $t$, scale: (a)-(b) $5 \mathrm{~ms} / \mathrm{div}$, (c)-(d) $10 \mathrm{~ms} / \mathrm{div}$, for time waveforms; Horizontal axis $f$, scale: $250 \mathrm{~Hz} /$ div, for spectra; Vertical axis $v_{c 2}$, scale : $200 \mathrm{mV} /$ div, for trajectories; Vertical axis $v_{\mathrm{c} 1}$, scale: $2 \mathrm{~V} / \mathrm{div}$, for time waveforms; Vertical axis $v_{\mathrm{c},}$, scale $: 10 \mathrm{~dB} / \mathrm{div}$, for spectra. Parameter values: $C_{1}\left(v_{c}\right)=23.60 \mathrm{nF}, C_{2}\left(v_{c}\right)=368.6 \mathrm{nF}, R\left(v_{c}\right)=1.533 \mathrm{k} \Omega, R_{0}\left(v_{c}\right)=$ $6.32 \Omega, L\left(v_{c}\right)=38.08 \mathrm{mlH}$ (with inherent series resistance $28.65 \Omega$ ), $G_{a}=-0.72 \mathrm{mS}, G_{b}=$ $-0.5 \mathrm{mS}$. (a) $v_{\mathrm{c}}=3.847 \mathrm{~V},+E=+0.65 \mathrm{~V},-E=-1 \mathrm{~V}$ 

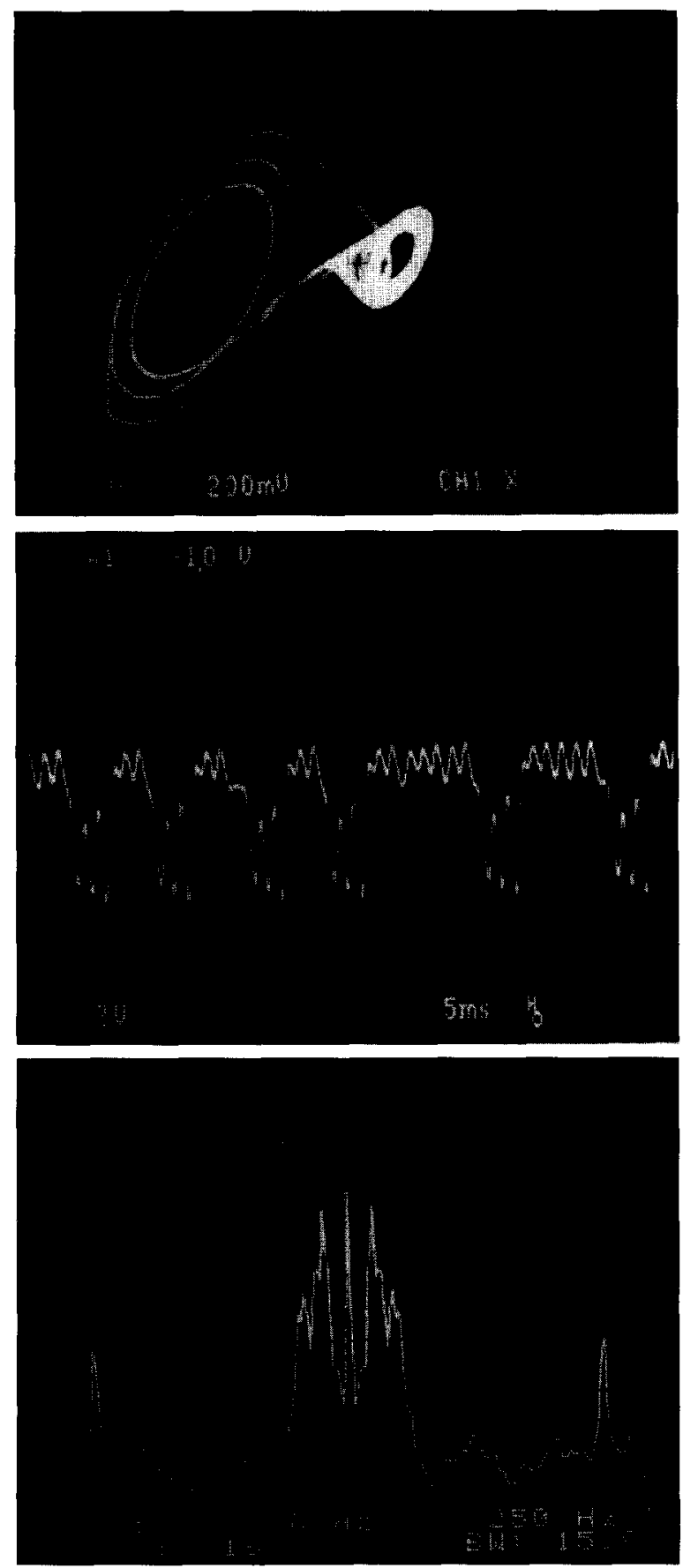

FiG. 15(b) $v_{c}=2.415 \mathrm{~V},+E=+0.35 \mathrm{~V},-E=-1 \mathrm{~V}$. 
G.-Q. Zhong et al.
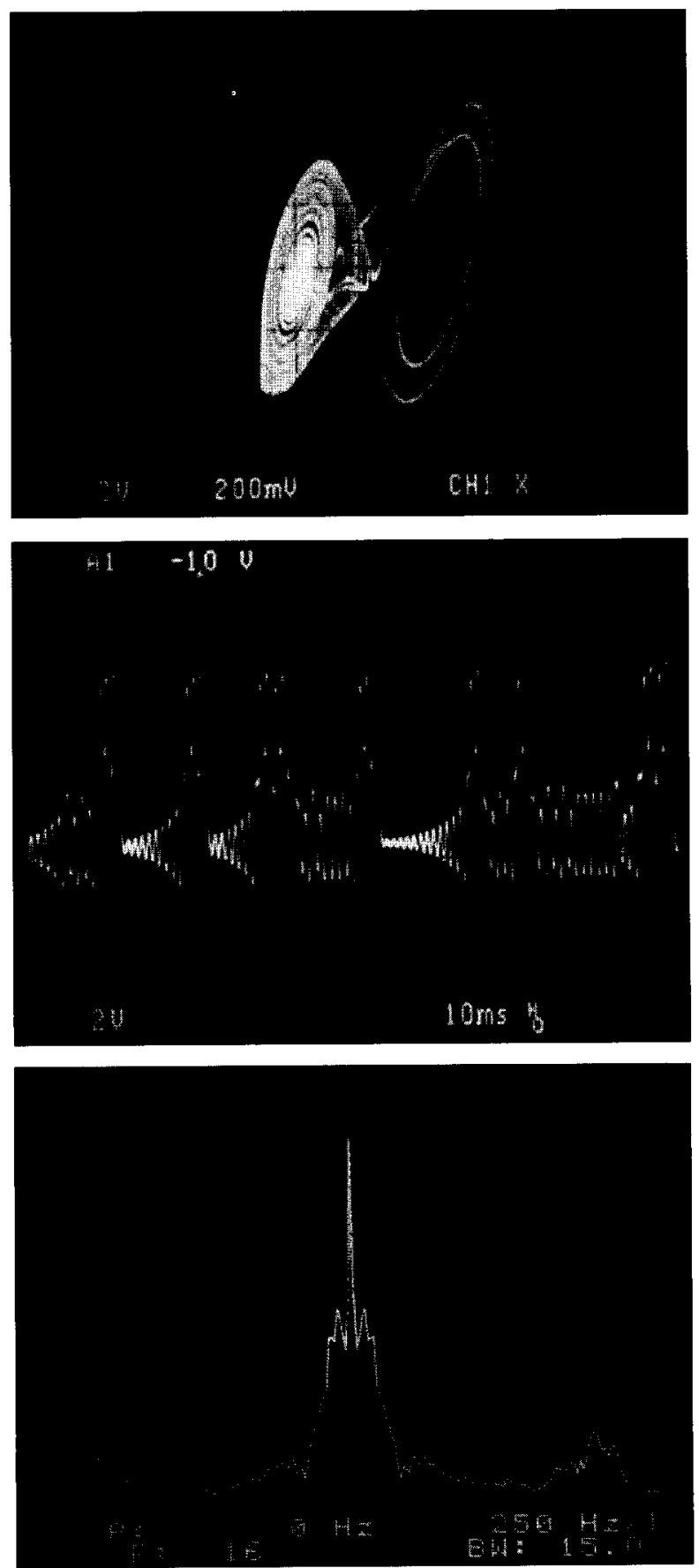

FIG. 15(c) $v_{\mathrm{c}}=-5.050 \mathrm{~V},+E=+1 \mathrm{~V},-E=-0.85 \mathrm{~V}$. 

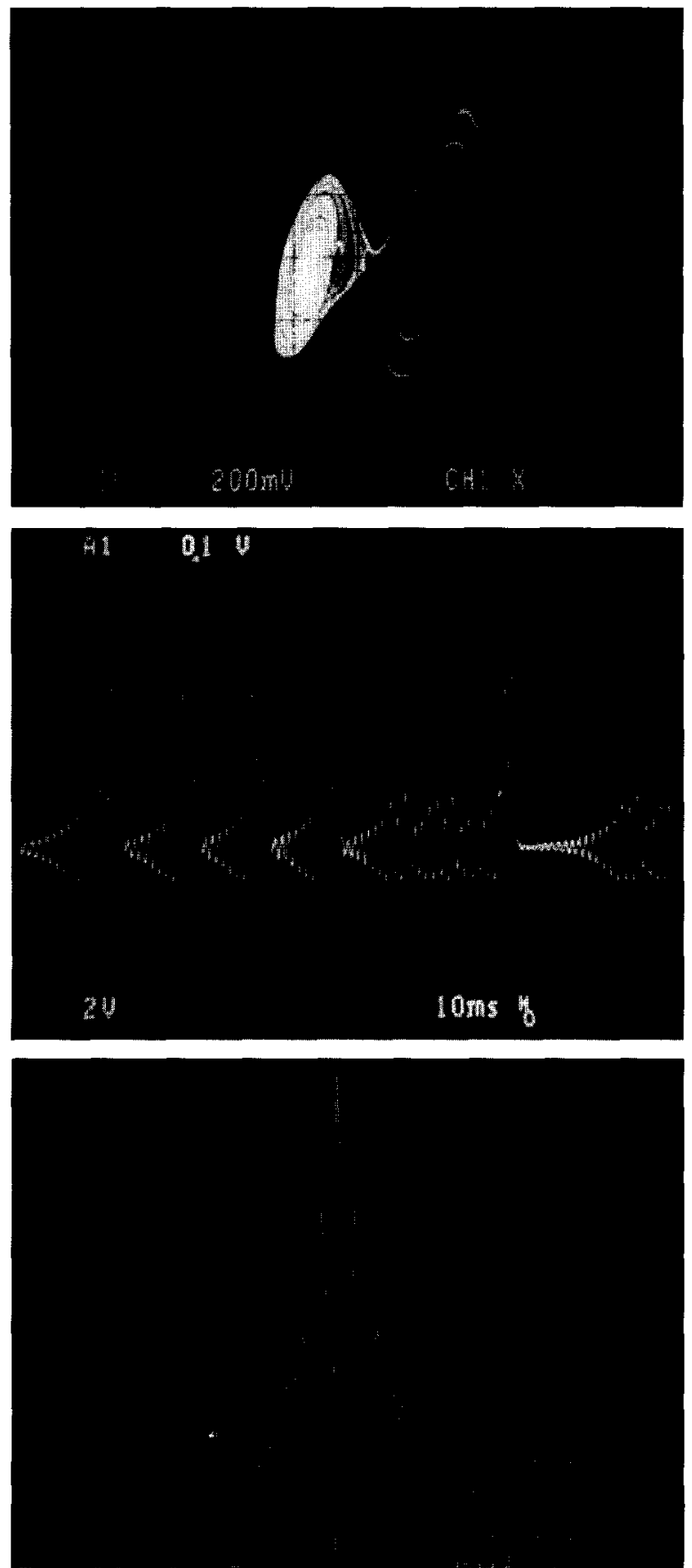

FIG. 15 (d) $v_{c}=-4.385 \mathrm{~V},+E=+1 \mathrm{~V},-E=-0.65 \mathrm{~V}$. 
circuit presented in this paper to generate signals for compositions and performances.

\section{Acknowledgements}

This work was supported in part by the Office of Naval Research under Grant N0001489-J-1402 and by the National Science Foundation under Grant MIP 86-14000. G. Q. Zhong would like to thank L. O. Chua for his support and valuable suggestion, I. Choi for specification discussions and utilizing this model to realize successful performances during Expo '93 in Seoul and Taejon, Korea, and G. Mayer-Kress, B. Shi, C. W. Wu, and L. Pivka for helpful discussions. R. Bargar would like to thank A. Jackson and L. Arsenault for discussions of digital simulations of Chua's circuit, and X. Rodet for assistance with the real-time circuit simulation and the interface shown in Fig. 10.

\section{References}

(1) M. J. Ogorzalek, "Taming chaos: Part II-Control", IEEE Trans. Circuits \& Syst., Vol. CAS-40, pp. 700-706, 1993.

(2) G. Chen and X. Dong, "From chaos to order-Perspectives and methodologies in controlling chaotic nonlinear dynamical systems", Int. J. Bifurcations Chaos, Vol. 3 , pp. 1363-1409, 1993.

(3) G. A. Johnson, T. E. Tigner and E. R. Hunt, "Controlling chaos in Chua's circuit", J. Circuits Syst. Comput., Vol. 3, pp. 109-117, 1993.

(4) K. Murali and M. Lakshmanan, "Controlling of chaos in the driven Chua's circuit", J. Circuits Syst. Comput., Vol. 3, pp. 125-137, 1993.

(5) G. Chen and X. Dong, "Controlling Chua's circuit", J. Circuits Syst. Comput., Vol. 3, pp. 139-149, 1993.

(6) G. Genesio and A. Tesi, "Distortion control of chaotic systems: The Chua's circuit", J. Circuits Syst. Comput., Vol. 3, pp. 151-171, 1993.

(7) T. T. Hartley and F. Mossayebi, "Control of Chua's circuit", J. Circuits Syst. Comput., Vol. 3, pp. 173-194, 1993.

(8) T. Kapitaniak, Lj. Kocarev and L. O. Chua, "Controlling chaos without feedback and control signals", Int. J. Bifurcations Chaos, Vol. 3, pp. 459-468, 1993.

(9) L. O. Chua, "The genesis of Chua's circuit", Archiv Elektronik Ubertragungstechnik, Vol. 46, pp. 250-257, 1992.

(10) T. Matsumoto, "A chaotic attractor from Chua's circuit", IEEE Trans. Circuits \& Syst., Vol. CAS-31, pp. 1055-1058, 1984.

(11) G. Q. Zhong and F. Ayrom, "Experimental confirmation of chaos from Chua's circuit", Int. J. Circuit Theory Appl., Vol. 13, pp. 93-98, 1985.

(12) G. Q. Zhong and F. Ayrom, "Periodicity and chaos in Chua's circuit", IEEE Trans. Circuits \& Syst., Vol. CAS-32, pp. 501-503, 1985.

(13) T. Matsomoto, L. O. Chua and R. Tokunaga, "Chaos via torus breakdown", IEEE Trans. Circuits \& Syst., Vol. CAS-34, pp. 240-253, 1987.

(14) T. S. Parker and L. O. Chua, "The dual double scroll equation", IEEE Trans. Circuits \& Syst., Vol. 34, pp. 1059-1073, 1987.

(15) S. Wu, "Chua's circuit family", Proc. IEEE, Vol. 75, pp. 1022-1032, 1987.

(16) P. Bartissol and L. O. Chua, "The double hook", IEEE Trans. Circuits \& Syst., Vol. CAS-35, pp. 1512-1522, 1988.

(17) C. P. Silva and L. O. Chua, "The overdamped double scroll family", Int. J. Circuit Theory Appl., Vol. 16, pp. 223-302, 1988. 
(18) L. O. Chua and G. N. Lin, "Canonical realization of Chua's circuit family", IEEE Trans. Circuits \& Syst., Vol. CAS-37, pp. 885-902, 1990.

(19) L. O. Chua, "Global unfolding of Chua's circuits", IEICE Trans. Fund. Electron. Comm. Comput. Sci., Vol. E76-A, pp. 704-734, 1993.

(20) F. Ayrom and G. Q. Zhong, "Chaos in Chua's circuit", Proc. IEE., Pt. D, Vol. 133, pp. 307-312, November 1986.

(21) X. Rodet, "Sound and music from Chua's circuit", J. Circuits Syst. Comput., Vol. 3, pp. 49-61, March 1993.

(22) G. Mayer-Kress, I. Choi, N. Weber, R. Bargar and A. Hübler, "Musical signals from Chua's circuit", IEEE Trans. Circuits \& Syst.—II : Analog Digital Signal Processing, Vol. CAS-40, pp. 688-695, 1993.

(23) 1. Choi, "A chaotic oscillator as a musical signal generator in an interactive performance system", in J. New Music Res., Ghent, Belgium, to appear.

(24) R. Bargar, I. Choi, S. Das and C. Goudeseune, "Model-based interactive sound for an immersive virtual environment", in "Proc. Int. Comput. Music Conf.", Aarhus, Denmark, September 1994.

(25) G. Mayer-Kress, I. Choi and R. Bargar, "Sound synthesis and music composition using Chua's oscillator", in "Proc. Int. Symp. Nonlinear Theory Appl. (Hawaii)", Vol. 5 (workshop), pp. 65-70, 1993.

(26) M. P. Kennedy, "Robust op amp realization of Chua's circuit", Frequenz, Vol. 46, pp. 66-80, 1992.

(27) A. Freed, "Tools for rapid prototyping of music sound synthesis algorithms and control strategies," in "Proc. Int. Comput. Music Conf.", San Jose, CA, October 1992.

(28) D. Wessel, "Timbre space as a musical control structure", Comput. Music J., Vol. 3, pp. 45-52, 1979.

(29) International MIDI Association, "MIDI 1.0 Specification", North Hollywood : International MIDI Association, 1983.

(30) G. Loy, "Musicians make a standard: The MIDI phenomenon", Comput. Music J., Vol. 9, pp. 8-26, 1985.

(31) N. Weber, CCSR, University of Illinois, private communication, September 1993.

(32) I. Choi, "anti-Odysseus: The Irreversibility of Time", composition for interactive performance with Chua's circuit, created in Center for New Music and Audio Technology (CNMAT) at U. C. Berkeley and Numerical Laboratory at National Center for Supercomputing Application (NCSA), University of Illinois at UrbanaChampaign, Premiered in World Expo, Taejon and Seoul, Korea, $20-23$ October, 1993.

\section{Appendix}

1. Voltage-controlled capacitor $C\left(v_{c}\right)$

The equivalent circuit of the voltage-controlled capacitor in Fig. 3 is shown in Fig. A(1). By applying Kirchhoff's Voltage Law (KVL) to the circuit shown in Fig. A(1), we have

$$
i=C \frac{\mathrm{d}\left(v-\frac{v v_{c}}{10 V}\right)}{\mathrm{d} t}=C\left(1-\frac{v_{c}}{10 V}\right) \frac{\mathrm{d} v}{\mathrm{~d} t}
$$

Let $C\left(v_{c}\right)$ be the equivalent capacitance between the two terminals. Then 
G.-Q. Zhong et al.

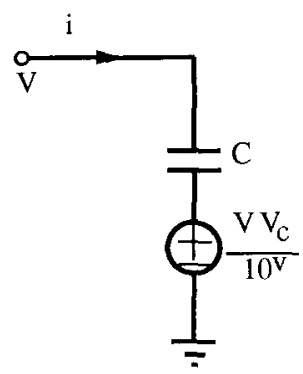

FIG. A(1). The equivalent circuit of voltage-controlled capacitor.

$$
i=C\left(v_{c}\right) \frac{\mathrm{d} v}{\mathrm{~d} t},
$$

i.e.

$$
C\left(v_{c}\right)=C\left(1-\frac{v_{c}}{10 V}\right)
$$

2. Voltage-controlled inductor $L\left(v_{c}\right)$

The equivalent circuit of the voltage-controlled inductor in Fig. 4 is shown in Fig. A(2). By applying KVL to the equivalent circuit in Fig. A(2), we have

$$
L \frac{\mathrm{d} i}{\mathrm{~d} t}=v-\frac{v v_{c}}{10 V}=v\left(1-\frac{v_{c}}{10 V}\right)
$$

Let $L\left(v_{c}\right)$ be the equivalent inductance between the two terminals, i.e.

$$
v=L\left(v_{c}\right) \frac{\mathrm{d} i}{\mathrm{~d} t} .
$$

Thus we obtain

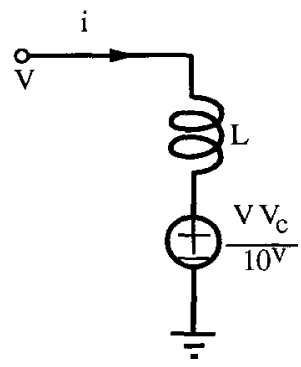

FIG. A(2). The equivalent circuit of voltage-controlled inductor. 


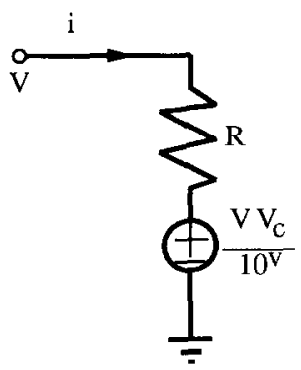

FIG. A(3). The equivalent circuit of voltage-controlled resistor.

$$
L\left(v_{c}\right)=\frac{L}{1-\frac{v_{c}}{10 V}} .
$$

3. Voltage-controlled resistor $R\left(v_{c}\right)$

The equivalent circuit of the voltage-controlled resistor in Fig. 5 is shown in Fig. A(3). By applying KVL to the equivalent circuit in Fig. A(3), we have

$$
i=\frac{1}{R}\left[v-\frac{v v_{c}}{10 V}\right]=\frac{v}{R}\left(1-\frac{v_{c}}{10 V}\right) .
$$

Substituting $v=i R\left(v_{c}\right)$ for $v$ above gives

$$
R\left(v_{c}\right)=\frac{R}{1-\frac{v_{c}}{10 V}} .
$$

\section{Voltage-controlled resistor with floating terminals}

The equivalent circuit of the voltage-controlled resistor with floating terminals in Fig. 6 , is shown in Fig. A(4).

By applying KVL to the equivalent circuit, we have

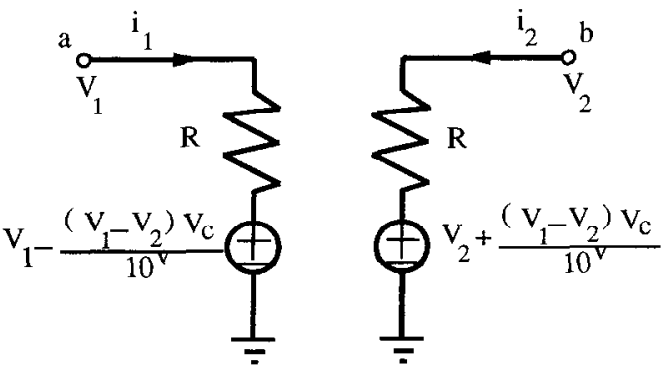

FIG. A(4). The equivalent circuit of voltage-controlled resistor with floating terminals. 
G.-Q. Zhong et al.

$$
\begin{gathered}
i_{1}=\frac{v_{1}-\left(v_{1}-\Delta v \frac{v_{c}}{10 V}\right)}{R}=\frac{\Delta v \frac{v_{c}}{10 V}}{R}, \\
i_{2}=\frac{v_{2}-\left(v_{2}+\Delta v \frac{v_{c}}{10 V}\right)}{R}=-\frac{\Delta v \frac{v_{c}}{10 V}}{R},
\end{gathered}
$$

where $\Delta v=v_{1}-v_{2}$.

Let $R_{1}\left(v_{c}\right)$ and $R_{2}\left(v_{c}\right)$ denote the voltage-controlled resistances for left branch and right branches of the equivalent circuit shown in Fig. A(4), respectively. Then we have

$$
\begin{gathered}
v_{1}-i_{1} R_{1}\left(v_{c}\right), \\
v_{2}=i_{2} R_{2}\left(v_{c}\right), \\
v_{1}-v_{2}=\Delta v=i_{1} R_{1}\left(v_{c}\right)-i_{2} R_{2}\left(v_{c}\right)=\frac{\Delta v \frac{v_{c}}{10 V}}{R}\left[R_{1}\left(v_{c}\right)+R_{2}\left(v_{c}\right)\right] .
\end{gathered}
$$

Thus the voltage-controlled resistance $R\left(v_{c}\right)$ with floating terminals above ground between terminals $a$ and $b$ is

$$
R\left(v_{c}\right)=R_{1}\left(v_{c}\right)+R_{2}\left(v_{c}\right)=\frac{R}{\frac{v_{c}}{10 V}} .
$$

